\title{
Joint inversion methods with relative density offset correction for muon tomography and gravity data, with application to volcano imaging
}

\author{
Peter G. Lelièvre ${ }^{\oplus},{ }^{1}$ Anne Barnoud ${ }^{\oplus}, 2,3$ Valentin Niess, ${ }^{2}$ Cristina Cârloganu, ${ }^{2}$ \\ Valérie Cayol $^{3,4}$ and Colin G. Farquharson ${ }^{1}$ \\ ${ }^{1}$ Memorial University of Newfoundland, St.John's, NL, A1B3X5, Canada.E-mail: plelievre@mun.ca \\ ${ }^{2}$ Université Clermont Auvergne, CNRS/IN2P3, LPC, F-63000 Clermont-Ferrand, France \\ ${ }^{3}$ Université Clermont Auvergne, CNRS, IRD, OPGC, Laboratoire Magmas et Volcans, F-63000 Clermont-Ferrand, France \\ ${ }^{4}$ Université de Lyon, Laboratoire Magmas et Volcans, UJM-UCA-CNRS-IRD, F-42023 Saint-Étienne, France
}

Accepted 2019 May 30. Received 2019 May 8; in original form 2018 December 12

\begin{abstract}
S UMMAR Y
Muography is a relatively new geophysical imaging method that uses muons to provide estimates of average densities along particular lines of sight. Muography can only see above the horizontal elevation of the detector and it is therefore attractive to attempt a joint inversion of muography data with gravity data, which is also responsive to density but generally requires combination with another geophysical data set to overcome issues related to non-uniqueness and poor depth resolution. Some previous work has investigated this joint inverse problem and demonstrated the potential improvements to be gained by jointly inverting muography and gravity data. However, there has yet to be a thorough investigation of different numerical approaches for formulating the joint inverse problem. Particularly important is how to account for the fact that even though the two data types are sensitive to the same physical quantity, density, they respond through different response functions. Moreover, the two measurements are affected by different systematic uncertainties that are difficult to model. In this work, we considered an approximation where the two density quantities, inferred from the two data types, can be related by an unknown scalar offset. We considered various existing and new joint inversion methods that might solve this problem and we applied them to a synthetic volcano imaging scenario based on the Puy de Dôme volcano in the Central Massif region of France. We used unstructured meshes in our modelling to adequately honour the significant topography in that scenario. Our experiments indicated that the most successful joint inversion method for this type of geological scenario was one in which the data misfit function is reformulated to automatically determine the best-fitting offset following a least-squares minimization argument. However, other approaches showed merit and we suggest several of the investigated methods be applied and compared for any specific joint inversion scenario.
\end{abstract}

Key words: Gravity anomalies and Earth structure; Inverse theory; Joint inversion; Numerical modelling; Tomography; Remote sensing of volcanoes.

\section{INTRODUCTION}

Muography is a natural source imaging method that uses muons, subatomic particles generated through interactions between cosmic particles and the Earth's atmosphere. The number of muons transmitted through a body of rock is, to first order, inversely related to the integrated density (total mass) along the path. Muons are counted as they pass through a muon detector, which provides direction information and, after processing, estimates of average densities along particular lines of sight. Several muographic images can then be combined to perform muon tomography, which involves 3-D density reconstruction from muographic data. In this sense, muon tomography might be compared to seismic tomography with straight rays.

Muography is a relatively new imaging method to be employed in geophysics. Atmospheric muons, produced high in the atmosphere, travel downwards at some angle before reaching the detector. Therefore, muography can effectively only see upwards from the detector, 
that is, above the horizontal elevation of the detector. Hence, geophysical studies using muography have typically involved targets with large and steep topographic relief, for example, human-built pyramids (e.g. Tanaka et al. 2010; Morishima et al. 2017) and volcanos (e.g. Cârloganu et al. 2013), but some work suggests the feasibility of deploying muon detectors inside mine workings or even boreholes (e.g. Liu et al. 2012; Schouten \& Ledru 2018).

Collection of muography data in the field is usually limited by cost, land and power access, and time. The required exposition time increases with an object's thickness and density; for objects less than a kilometre thick and small muon telescopes $\left(\sim 1 \mathrm{~m}^{2}\right)$, data are typically recorded over several months at each detector location. Because of those survey considerations, and the limitation of muography data to only see upwards from the detector, it is attractive to attempt a joint inversion of muography data with another complimentary geophysical data type. A clear choice is gravity, which is responsive to the same physical property, density.

Some previous work has investigated joint inversion of those two data types through either synthetic (Davis \& Oldenburg 2012; Jourde et al. 2015; Barnoud et al. 2019) or both synthetic and real data studies (Nishiyama et al. 2014, 2017; Rosas-Carbajal et al. 2017). Those studies demonstrated the potential improvements to be gained by jointly inverting muography and gravity data. However, there has yet to be a thorough investigation of different numerical approaches for formulating the joint inverse problem to account for the fact that the two data sets respond to density through different mathematical response functions, which results in some generally unknown offset. The work of Davis \& Oldenburg (2012), Jourde et al. (2015), Nishiyama et al. (2017) and Barnoud et al. (2019) considered joint inversion methods that assumed both data sets were responsive to the same density quantity, which requires an accurate manual relative shifting of the two data sets prior to inversion, for example, as guided by prior information. Instead of relying on such prior information, Rosas-Carbajal et al. (2017) included an additional scalar model parameter in the inversion representing the unknown relative density offset.

In this paper we first explain the basics of forward modelling for muography and gravity data. We then develop the mathematics for various joint inversion approaches, with the primary goal of assessing the relative offset between the two density quantities. We test our methods on a volcano imaging problem based on the Puy de Dôme volcano in the Central Massif region of France. While the test scenario is synthetic, it uses a fully 3-D model of the volcano built from existing geological and geophysical information, and it includes data measurement locations at which preliminary field survey data has already been collected.

\section{METHODS}

\subsection{Forward modelling}

We discretize the Earth volume of interest into a 3-D unstructured mesh of space-filling tetrahedra. Rectilinear meshes could also be used but we chose unstructured meshes for this work because they are better able to honour complicated a priori surfaces, for example significant topography, without requiring large numbers of mesh cells. To calculate the gravity response of a density model, we use the methods of Okabe (1979) (see also Lelièvre et al. 2012).

We now provide a layperson's description of the phenomena that control the path and scattering of the subatomic particles involved in muography. Please refer to Nagamine et al. (1995), Procureur
(2018) and Tanabashi et al. (2018) for more details. Raw muography data comprises muon hits against different panels in the muon detector assembly. From this information, one can separate the high energy muons that propagated straight through the imaging target (volcano for example), which carry the density information, from all the other charged particles that give signals in the detector. The physical phenomena that control the subatomic particles as they travel through the atmosphere and target are stochastic processes. Details on the production of atmospheric muons and their energy loss can be found in Tanabashi et al. (2018). The muon transport problem can be efficiently solved by Monte-Carlo simulation, for example as described in Niess et al. (2018a). After various processing steps (see Niess et al. 2018b), one arrives at the final muography data that will be input into the geophysical inversion; that data comprises average densities along solid angles, by which we mean small bins across the azimuthal and polar angles, for example in one degree increments.

The mathematics of the forward modelling solution required is relatively simple compared to the stochastic physical modelling that provides the average density estimates. Given a modelling mesh (a discretization of the Earth volume of interest) and the detector location, one must integrate the densities in the mesh across each solid angle bin corresponding to each muography data value (each average density):

$\rho_{\mathrm{av}}=\frac{\int_{\Omega} \int_{r} \rho \mathrm{d} r \mathrm{~d} \Omega}{\int_{\Omega} \int_{r} \mathrm{~d} r \mathrm{~d} \Omega}$,

where

(1) $\rho_{\mathrm{av}}$ is the average density

(2) $\Omega$ is the solid angle bin

(3) $r$ is distance from the detector

(4) $\rho$ is absolute density.

The numerator in eq. (1) is the integrated density (total mass) in the volume of the mesh subtended by the solid angle. The denominator divides by the volume to provide the average density.

To numerically integrate eq. (1) we utilize straight ray tracing, for example as would be used for a seismic tomography problem. If rays are traced across a grid of angles that subdivide the solid angle $\Omega$ then we are in essence calculating eq. (1) numerically in a spherical coordinate system:

$\rho_{\mathrm{av}}=\frac{\sum_{i=1}^{A} \sum_{j=1}^{P} \sum_{k=1}^{R} \rho_{k} d_{k} \delta \Omega}{\sum_{i=1}^{A} \sum_{j=1}^{P} \sum_{k=1}^{R} d_{k} \delta \Omega}$,

where

(1) $\delta \Omega$ is a sub-bin, a division of the solid angle $\Omega$

(2) the first sum is over the azimuthal angles that subdivide the solid angle $\Omega$

(3) the second sum is over the polar angles that subdivide the solid angle $\Omega$

(4) the third sum is over the cells in the mesh encountered by a ray traced along the centre of a particular solid angle sub-bin $\delta \Omega$

(5) $\rho_{k}$ is the density in one of the traced cells

(6) $d_{k}$ is the distance that the traced ray passed through the cell.

The procedure is therefore to trace each ray along the centre of each solid angle sub-bin to determine the cells encountered and then calculate the sums above. We can reduce the numerical error by increasing the number of sub-bins in the azimuthal and polar directions that subdivide each solid angle $\Omega$. However, because of the geometrical spreading of the rays away from a muon detector, the 
errors in this calculation increase with distance from the detector. Therefore, the number of sub-bins required to adequately reduce the modelling error must be determined by the size of the cells on the far side of the mesh from the detector.

\subsection{Joint inversion for a single density model for both data types}

\subsubsection{Deterministic inversion}

The term joint inversion has been used in many different ways in the literature (see Moorkamp et al. 2016). Here we use the term to denote any inversion that simultaneously uses two types of geophysical data. In this work we formulated the joint inverse problem in many ways, all of them as deterministic formulations where we minimized an objective function using a Gauss-Newton minimization algorithm. The simplest form of the objective function that we considered was

$\Phi=\lambda_{1} \Phi_{d 1}(\mathbf{m})+\lambda_{2} \Phi_{d 2}(\mathbf{m})+\Phi_{m}(\mathbf{m})$,

where

(1) $\Phi$ is the total objective function to be minimized

(2) $\mathbf{m}$ is a model vector containing the density values in each cell of the inversion mesh

(3) $\Phi_{d 1}$ measures the misfit between the observed gravity data and the gravity response of the candidate model $\mathbf{m}$

(4) $\Phi_{d 2}$ measures the misfit between the observed muography data and the muography response of the candidate model

(5) $\Phi_{m}$ is a regularization term, referred to in the literature as a 'model objective' or 'model norm' term, that measures the amount of structure in the candidate model

(6) $\lambda_{1}$ and $\lambda_{2}$ are termed 'trade-off parameters' because changing them represents a trade-off between the two goals of an inversion: recover a model that fits the data and that contains geologically realistic features.

For most inversions we used the standard data misfit form

$\Phi_{d}=\frac{1}{N} \sum_{i=1}^{N} \frac{\left(d_{i}^{\mathrm{pred}}-d_{i}^{\mathrm{obs}}\right)^{2}}{\sigma_{i}^{2}}$

where

(1) $N$ is the number of data observations

(2) $d_{i}^{\text {obs }}$ are the observed data measurements

(3) $d_{i}^{\text {pred }}$ are the predicted data synthesized for the candidate model

(4) $\sigma_{i}$ are uncorrelated uncertainties assigned to the observed data.

For some inversions we altered the form of the data misfit that will be discussed later.

Both the gravity and muography forward problems can be written as

$\mathbf{d}^{\text {pred }}=\mathbf{G m}$,

where

(1) $\mathbf{d}^{\text {pred }}$ is a vector containing all the predicted (synthetized) data

(2) $\mathbf{G}$ is a linear matrix operator usually termed the 'sensitivity matrix'.

The element in the $i$ th row and $j$ th column of $\mathbf{G}$ is the contribution of the density in the $j$ th mesh cell to the $i$ th data value.
Our model norm term, $\Phi_{m}$, can contain a smallness and smoothness component:

$\Phi_{m}=\alpha_{s} \sum_{j=1}^{M} w_{s, j} m_{j}^{2}+\alpha_{m} \sum_{k=1}^{F} w_{m, k}\left(m_{k-}-m_{k+}\right)^{2}$,

where

(1) $M$ is the number of cells in the mesh

(2) $F$ is the number of internal cell faces in the mesh

(3) $m_{j}$ is the density value in the $j$ th mesh cell

(4) $m_{k-}$ and $m_{k+}$ denote the cells adjacent to the $k$ th internal face in the mesh

(5) the weights $w_{s, j}$ and $w_{m, k}$ can be used to counteract the decay of the sensitivity kernels

(6) the scalar weights $\alpha_{s}$ and $\alpha_{m}$ can be set pre-inversion to increase or decrease the importance of the smallness and smoothness terms, respectively.

To compensate for the decay of the gravity data sensitivity kernels, we employed a distance weighting strategy following Li \& Oldenburg (2000) with a weighting power of 2.0 to account for the distance-squared fall-off of the gravity kernels:

$w_{j}=\left(\sum_{i=1}^{N}\left(\int_{\Delta V_{j}} \frac{\mathrm{d} v}{\left(R_{i j}+R_{0}\right)^{2}}\right)^{2}\right)^{1 / 2}$,

where

(1) $w_{j}$ is the distance weight applied to the $j$ th mesh cell

(2) $\Delta V_{j}$ is the volume of the $j$ th mesh cell

(3) $R_{i j}$ is the distance between the $i$ th observation location and a point within the $j$ th mesh cell

(4) $R_{0}$ is some small constant chosen to avoid numerical instability (possible division by zero).

For muography data, Rosas-Carbajal et al. (2017) used a distanceto-detector weighting. We took a similar approach and used the distance weighting strategy of Li \& Oldenburg (2000) with a weighting power of 2.0 to account for the geometrical spreading of the solid angles with distance away from the detectors.

We used a discrepancy principle approach to determine the tradeoff parameters $\lambda_{1}$ and $\lambda_{2}$ in eq. (3): we defined target misfits equal to one, which is the expected value for the $\chi^{2}$ misfit function in eq. (4), and we sought values of $\lambda_{1}$ and $\lambda_{2}$ that yield those targets. See Lelièvre et al. (2012) for the algorithmic details on how we determined appropriate values for the two trade-off parameters. This approach requires an accurate knowledge of data errors, and the problem formulation and algorithmic approach of Lelièvre et al. (2012) avoids the possibility of underfitting one data type and overfitting the other: both will be fit to their target misfits.

\subsubsection{Relative density offset correction}

The complication with using a single density model for both data types in a joint inversion, for example as in eq. (3), is that the two data sets are effectively responsive to different density quantities. Gravity data respond to relative density with respect to a background value associated with the Bouguer and terrain corrections. There are various methods that one can use to determine a reasonable background density (e.g. see Linsser 1965; Yamamoto 1999; Tontini et al. 2007) but one generally accepts that there is some uncertainty here. For muography data, the modelling methods that translate muon counts to average densities are generally biased towards lower 
average densities, caused by the diffusion of low-energy muons (Nishiyama et al. 2014; Rosas-Carbajal et al. 2017), but quantifying the bias is difficult. Hence, a systematic relative offset between the density distributions inferred from the two measurements must be taken into account. In this work we assumed that the relative offset could be approximated to first order as a single constant for all measurements; in practice, it may vary somewhat between measurements but we leave treatment of that more complicated scenario for future work. Below we discuss methods to estimate a constant relative offset.

\subsection{Methods to automatically estimate a constant offset}

\subsubsection{Automatic least-squares minimization for the offset}

For some of our inversions we considered an alteration to the standard misfit function in eq. (4). We added an additional scalar quantity, $c$, which is, in effect, removed from the observed data:

$\Phi_{d 2}=\frac{1}{N} \sum_{i=1}^{N} \frac{\left(d_{2, i}^{\mathrm{pred}}-d_{2, i}^{\mathrm{obs}}+c\right)^{2}}{\sigma_{2, i}^{2}}$.

We applied this in the data misfit term for the muography data only, simply because it is easier to do so there than in the gravity data term. For muography data, which are average densities integrated along solid angles, if one adds or subtracts a constant from the density model then the data response changes by that same constant, for all data values. To clarify, we write

$\mathbf{d}_{2}^{\text {pred }}=\mathbf{G}_{2}(\mathbf{m}+\mathbf{e} c)=\mathbf{G}_{2} \mathbf{m}+\mathbf{G}_{2} \mathbf{e} c=\mathbf{G}_{2} \mathbf{m}+\mathbf{e} c$,

where $\mathbf{e}$ is a vector of ones and $\mathbf{G}_{2} \mathbf{e}=\mathbf{e}$ because each row of $\mathbf{G}_{2}$ is normalized to sum to one (recall that muography data are average densities). However, for gravity data we have $\mathbf{G}_{1} \mathbf{e}=\mathbf{d}^{\text {unit }} \neq \mathbf{e}$ where $\mathbf{d}^{\text {unit }}$ is the response of a unit density filling the modelling mesh. That response is only constant if the mesh has infinite lateral dimensions and flat topography, and the data observations are on a horizontal surface, conditions that are not met in practice.

Returning to eq. (8), we propose to take a value of $c$ that minimizes the altered muography data misfit in a least-squares sense:

$c=-\frac{1}{N} \sum_{i=1}^{N}\left(d_{2, i}^{\mathrm{pred}}-d_{2, i}^{\mathrm{obs}}\right)=-\frac{1}{N} \mathbf{e}^{\mathrm{T}}\left(\mathbf{G}_{2} \mathbf{m}-\mathbf{d}_{2}^{\mathrm{obs}}\right)$,

which is the average data residual. The misfit in eq. (8) then becomes

$\Phi_{d 2}=\sum_{i=1}^{N} \frac{\left(d_{2, i}^{\mathrm{pred}}-d_{2, i}^{\mathrm{obs}}-\frac{1}{N} \sum_{i=1}^{N}\left(d_{2, i}^{\mathrm{pred}}-d_{2, i}^{\mathrm{obs}}\right)\right)^{2}}{\sigma_{2, i}^{2}}$,

or written in matrix-vector form we have

$$
\begin{aligned}
\Phi_{d 2} & =\left\|\mathbf{W}_{d 2}\left(\mathbf{G}_{2} \mathbf{m}-\mathbf{d}_{2}^{\mathrm{obs}}+\mathbf{e} c\right)\right\|^{2} \\
& =\left\|\mathbf{W}_{d 2}\left(\mathbf{G}_{2} \mathbf{m}-\mathbf{d}_{2}^{\mathrm{obs}}-\mathbf{e} \frac{1}{N} \mathbf{e}^{\mathrm{T}}\left(\mathbf{G}_{2} \mathbf{m}-\mathbf{d}_{2}^{\mathrm{obs}}\right)\right)\right\|^{2}, \\
& =\left\|\mathbf{W}_{d 2}\left(\mathbf{I}-\frac{1}{N} \mathbf{e}^{\mathrm{T}}\right)\left(\mathbf{G}_{2} \mathbf{m}-\mathbf{d}_{2}^{\mathrm{obs}}\right)\right\|^{2}
\end{aligned}
$$

where $\mathbf{W}_{d 2}$ is a diagonal matrix that holds the assigned uncertainties, $\sigma_{i}$, and $\mathbf{e}^{\mathrm{T}}$ multiplied by a vector sums the elements of that vector. Hence, the only change required to the inversion algorithm is to insert the operator $\left(\mathbf{I}-\frac{1}{N} \mathbf{e e}^{\mathrm{T}}\right)$ when calculating the muography data misfit. The effect is to effectively ignore a constant shift in the muography data, with the best-fitting shift determined automatically. We will refer to this automatic least-squares misfitreformulation approach as the 'LSM' approach in the remainder of this manuscript.

\subsubsection{Additional offset parameter in the inversion}

Another approach is to write the misfit as in eq. (8) but treat $c$ as an unknown scalar parameter in the inversion. For some of our inversions, we added a term to the objective function to penalize large values of $c$ :

$\Phi=\lambda_{1} \Phi_{d 1}(\mathbf{m})+\lambda_{2} \Phi_{d 2}(\mathbf{m}, c)+\Phi_{m}(\mathbf{m})+\alpha_{c} c^{2}$,

where the scalar weight $\alpha_{c}$ can be set pre-inversion to increase or decrease the importance of the additional term. This is in essence the approach of Rosas-Carbajal et al. (2017) but our numerical formulation is slightly different.

\subsection{Joint inversion for two density models}

Here, we dispense with the complication of having to find a relative offset by treating the two density models separately. With two models, we must add an additional term, $\Phi_{\text {joint }}$, to the objective function to encourage the two models to be similar:

$$
\begin{aligned}
\Phi=\lambda_{1} & \Phi_{d 1}\left(\mathbf{m}_{1}\right)+\lambda_{2} \Phi_{d 2}\left(\mathbf{m}_{2}\right) \\
& +\Phi_{m}\left(\mathbf{m}_{1}\right)+\Phi_{m}\left(\mathbf{m}_{2}\right) \\
& +\gamma \Phi_{\text {joint }}\left(\mathbf{m}_{1}, \mathbf{m}_{2}\right)
\end{aligned}
$$

where $\gamma$ is treated as a parameter to be heated (iteratively increased) following the approach of Lelièvre et al. (2012). Moorkamp et al. (2016) and Lelièvre et al. (2012) discuss some of the various 'coupling measures' for this purpose. We considered two coupling measures that assume linear relationships between the two density models.

\subsubsection{Coupling via an explicit linear relationship}

With enough prior information, one might assume an explicit linear relationship between the two models, $m_{1}$ and $m_{2}$ :

$m_{2}=a m_{1}+b$,

where $a$ and $b$ are some linear parameters. In the case of inverting gravity and muography data, we write $a=1$ and $b=c$ so

$m_{2}=m_{1}+c$

and a simple joint coupling measure is

$\Phi_{\text {joint }}=\left\|m_{1}-m_{2}+c\right\|^{2}$.

However, $c$ is treated as a prior here and, considering eq. (9), in the limit $\gamma \rightarrow \infty$ this formulation of the joint inverse problem becomes identical to a joint inversion for a single density model and some prior relative offset $c$ between the two data sets. Hence, we do not consider this coupling approach any further. However, the methods developed in the next subsection follow directly from eq. (16).

\subsubsection{Coupling via equal gradients}

If $\mathbf{D}$ is a spatial gradient or difference operator, for example one of the options mentioned by Lelièvre \& Farquharson (2013), then 
$\mathbf{D e}=\mathbf{0}$ (a vector of zeros) and from taking spatial derivatives of eq. (16) it follows that

$\mathbf{D m}_{1}=\mathbf{D m}_{2}$.

This effectively specifies that the two models have similar structure (equal spatial gradients). We can now dispense with any constant offset parameter $c$ and use the joint coupling measure

$\Phi_{\text {joint }}=\left\|\mathbf{D}\left(\mathbf{m}_{1}-\mathbf{m}_{2}\right)\right\|^{2}$.

For D we elected to use a simple difference operator that measures differences across neighbouring cells (see Günther et al. 2006; Lelièvre \& Farquharson 2013).

This approach allows us to specify an explicit linear relationship between the two density models with a slope of one and some unknown vertical axis intercept, which corresponds to the relative offset parameter. The cross-gradient coupling measure (e.g. Fregoso \& Gallardo 2009) could be applied here but that coupling uses spatial information only and is unable to specify anything about the linear relationship between the two density models. The power of the cross-gradient approach is in its general applicability but in our scenario we have powerful constraining prior information that can and should be used: the two density models are linearly related with a slope of one. Lelièvre et al. (2012) introduced a coupling measure for use when some linear relationship is assumed to exist but the exact linear parameters are unknown. Lelièvre et al. (2012) called this an 'implicit linear coupling' and used a cross-correlation from statistics. That coupling is only able to specify that the linear relationship between the two density models has a positive slope. The coupling measure that we propose in eq. (19) is based on the spatial gradient but should, in theory, provide a more forceful constraint than cross-gradient or implicit linear coupling.

\section{TESTS AND RESULTS}

\subsection{Puy de Dôme}

Puy de Dôme is a volcano located in the French Massif Central ancient volcanic zone. It belongs to the Chaîne des Puys, composed of about 80 monogenetic volcanoes built on a granitic basement. The Puy de Dôme is a $11000 \mathrm{yr}$ old trachytic dome, formed by two distinct extrusions (Boivin et al. 2017).

Puy de Dôme has been imaged using classical geophysical methods: ERT, gravimetry and magnetometry (Portal et al. 2013, 2016). Those methods have a rapidly decreasing sensitivity with depth. Muographic experiments have been conducted (Cârloganu et al. 2013; Ambrosino et al. 2015) with the potential of improving the imaging of structures inside the dome, hence improving our knowledge of the construction of this edifice.

The primary objective of this study is to determine which methods might be most helpful for consistently accounting for a scalar relative density offset when jointly inverting real gravity and muography survey data for Puy de Dôme or other volcanoes with roughly similar internal density structure. Within this scope, we test various methods on a synthetic model based on the expected internal structure of Puy de Dôme and real survey acquisition geometries.

\subsection{Building the Puy de Dôme model}

Four interpreted vertical cross-sections were taken from the work of Portal (2015) and Portal et al. (2016), inferred from gravimetric and magnetic data modelling. Those were digitized using the manual
Table 1. Absolute and relative densities (relative to $1.6 \mathrm{~g} \mathrm{~cm}^{-3}$ ) used for the three units in our synthetic model. Colours used in Figs 1 and 2 are in parentheses in the left column.

\begin{tabular}{lcc}
\hline Unit & $\begin{array}{c}\text { Absolute density } \\
\left(\mathrm{g} \mathrm{cm}^{-3}\right)\end{array}$ & $\begin{array}{c}\text { Relative density } \\
\left(\mathrm{g} \mathrm{cm}^{-3}\right)\end{array}$ \\
\hline Background (blue) & 1.6 & 0.0 \\
Volcanic dome (green) & 1.8 & 0.2 \\
Conduit (red) & 2.1 & 0.5 \\
\hline
\end{tabular}

model-building program FacetModeller (Lelièvre et al. 2018). The information in the cross-sections was interpolated throughout the 3-D volume of interest and combined with topography information from a digital elevation model. The various resulting model surfaces were sewn together into a watertight surface-based model using FacetModeller. Such a model represents a polygonal linear complex (PLC; see Miller et al. 1996; Si 2015) that can be passed into a meshing program to fill the volume of interest with tetrahedra while constraining the mesh to contain tetrahedral faces that honour the polygonal facets in the PLC. For that purpose we used TetGen ( $\mathrm{Si}$ 2017).

The interpreted cross-sections in Portal et al. (2016) consist of various layered extrusive units with other intrusive units passing vertically through them. For the purpose of testing the methods presented above, we simplified the model somewhat. First, the intrusive units outside the main dome were removed because they do not affect the muography response. Second, the gravity responses of the near-horizontal units are minimal so we ignored any density contrast between those. The result is an anomalously high density unit that we refer to as the 'volcanic dome', a higher density 'conduit' unit passing through the dome vertically, and the rest of the model taking the background density. The absolute densities used are listed in Table 1. Three perspective views of the model are shown in Fig. 1 and cross-sections through the model are shown in Fig. 2. To simplify those figures, and others that follow, we do not include axis labels. Instead, we provide the coordinate limits for the model in Table 2: the volume is $2.1 \mathrm{~km}$ by $2.1 \mathrm{~km}$ laterally by approximately $660 \mathrm{~m}$ vertically.

\subsection{Gravity data}

The 648 gravity observation locations were taken from a series of real ground surveys undertaken on Puy de Dôme over several years (Portal et al. 2013, 2016). They were placed $0.1 \mathrm{~m}$ above the topography surface. We calculated the gravity response with respect to a background density value of $1.6 \mathrm{~g} \mathrm{~cm}^{-3}$. We added noise (synthetic measurement errors) from a normal distribution with standard deviation equal to $0.039 \mathrm{mGal}$ and mean of zero, which corresponds to the estimated average noise level in the ground survey data collected. Following Bijani et al. (2017), we made sure that

$\left|1-\frac{1}{N} \sum_{j=1}^{N}\left(\frac{\epsilon_{j}}{\sigma_{j}}\right)^{2}\right| \leq \tau$,

where

(1) $\epsilon_{j}$ is the noise added to the $j$ th data observation

(2) $\sigma_{j}$ is the standard deviation of the related normal distribution

(3) $\tau$ is some small tolerance; we used 0.01 .

This means that the theoretical expected value of one for the $\chi^{2}$ misfit in eq. (4) was consistent with the actual noise added 
(a)

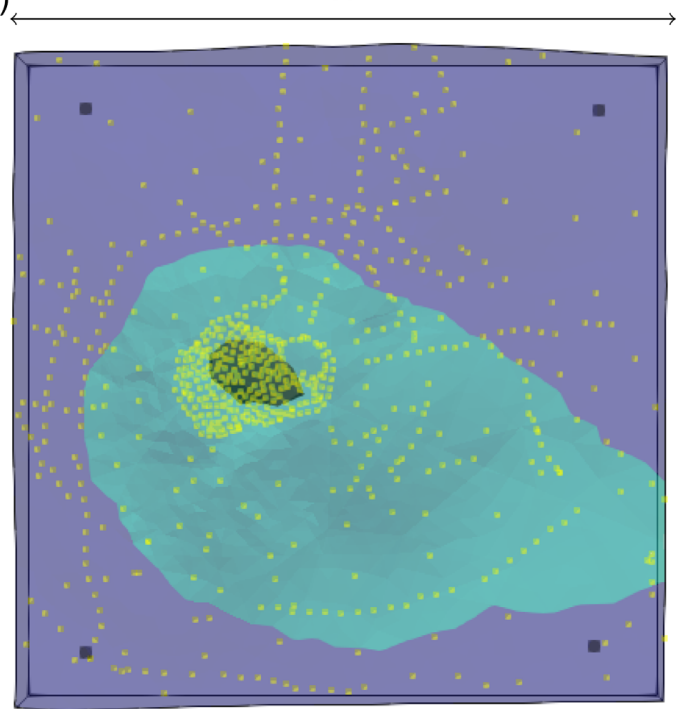

(b)

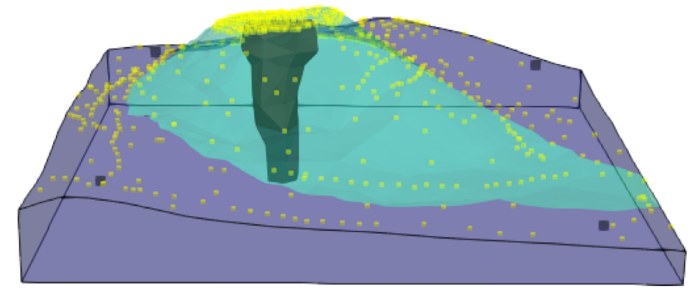

(c)

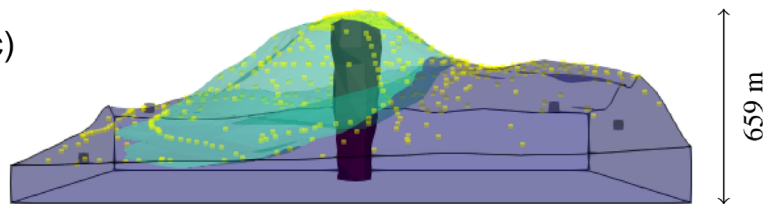

Figure 1. Three perspective views of the Puy de Dôme model used for our synthetic modelling tests: (a) from overhead; (b) from the south; (c) from the east. The model is semi-transparent to show the different units inside the volume: the conduit is red, the volcanic dome green and the background blue. The outline of the modelling region is drawn with black lines. The small yellow dots indicate the location of gravity data and larger black squares indicate the location of the four muon detectors.

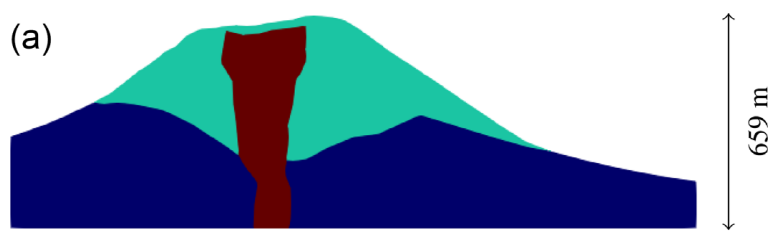

$2100 \mathrm{~m}$

(b)

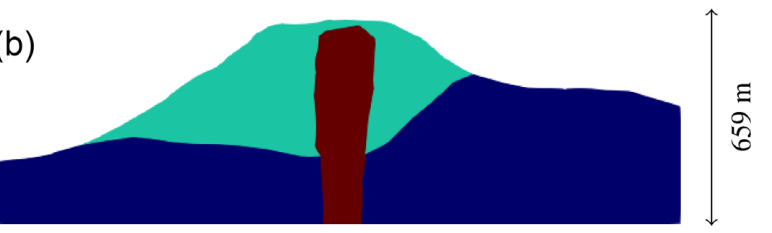

$2100 \mathrm{~m}$

Figure 2. Cross-sections through the Puy de Dôme model used for our synthetic modelling tests: (a) a west-east section at northing $19170 \mathrm{~m}$, viewed from the south (west at left); (b) a south-north section at easting $7080 \mathrm{~m}$, viewed from the east (south at left). These cross-sections pass through the approximate lateral centre of the conduit.
Table 2. Coordinate limits for our synthetic model volume in our local coordinate system.

\begin{tabular}{lccc}
\hline Direction & Minimum $(\mathrm{m})$ & Maximum $(\mathrm{m})$ & Range $(\mathrm{m})$ \\
\hline Easting & 6300 & 8400 & 2100 \\
Northing & 18100 & 20200 & 2100 \\
Elevation & 800 & 1459 & 659 \\
\hline
\end{tabular}

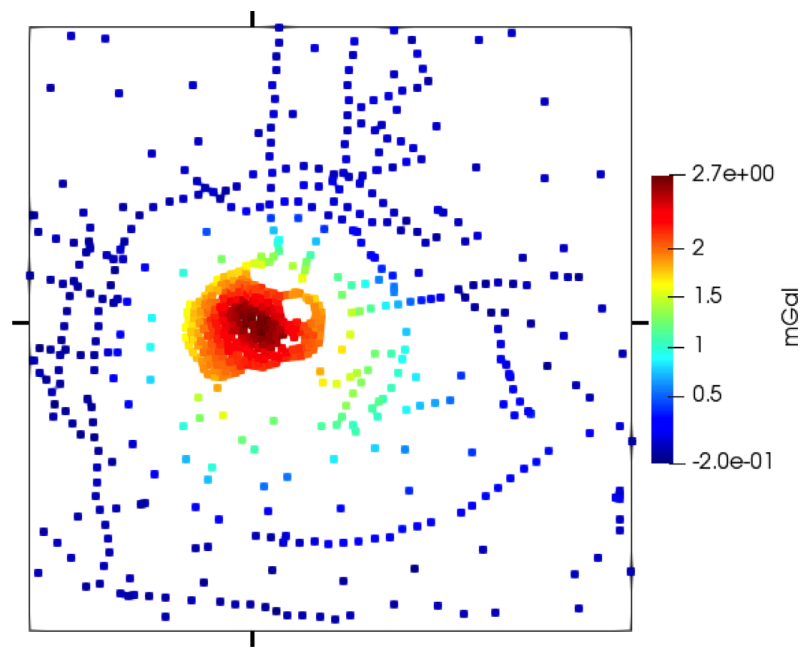

Figure 3. An overhead view of the noisy synthetic gravity data plotted as coloured points at their observation locations. The colour bar shows the gravity response in $\mathrm{mGal}$. The large black bounding square corresponds to the outline of the modelling volume. The small black marks on that bounding square indicate the locations of the cross-sections in Fig. 2.

and was therefore legitimate for analysing the synthetic inversion results. The resulting noisy synthetic gravity data are shown in Fig. 3.

\subsection{Muography data}

For the muography data, we used four muon detector locations. One of those locations was used in the field for preliminary campaigns on Puy de Dôme. The three others were selected to provide adequate illumination of the dome from different angles. Jourde et al. (2015) suggested that gravity data may not significantly improve the joint inversion results when several muon detectors already provide excellent coverage of the geological target. However, their work also suggests that if the muography data can recover the true density distribution in the upper dome then the joint inversion may enable the gravity data to better recover the true model at depth, below the coverage of the muography data.

All detectors were treated as points located $1.0 \mathrm{~m}$ above the topography surface. For each detector we used solid angle bins every one degree and only used those bins that intersected the model. We used the spherical integration framework with each solid angle bin divided into 64 sub-bins (eight in each angular direction). This provided 3400 muography data measurements. Fig. 4 shows the calculated muography data (average densities).

For muography data, generating synthetic noise that is consistent with all the relevant physical phenomena comes with significant 

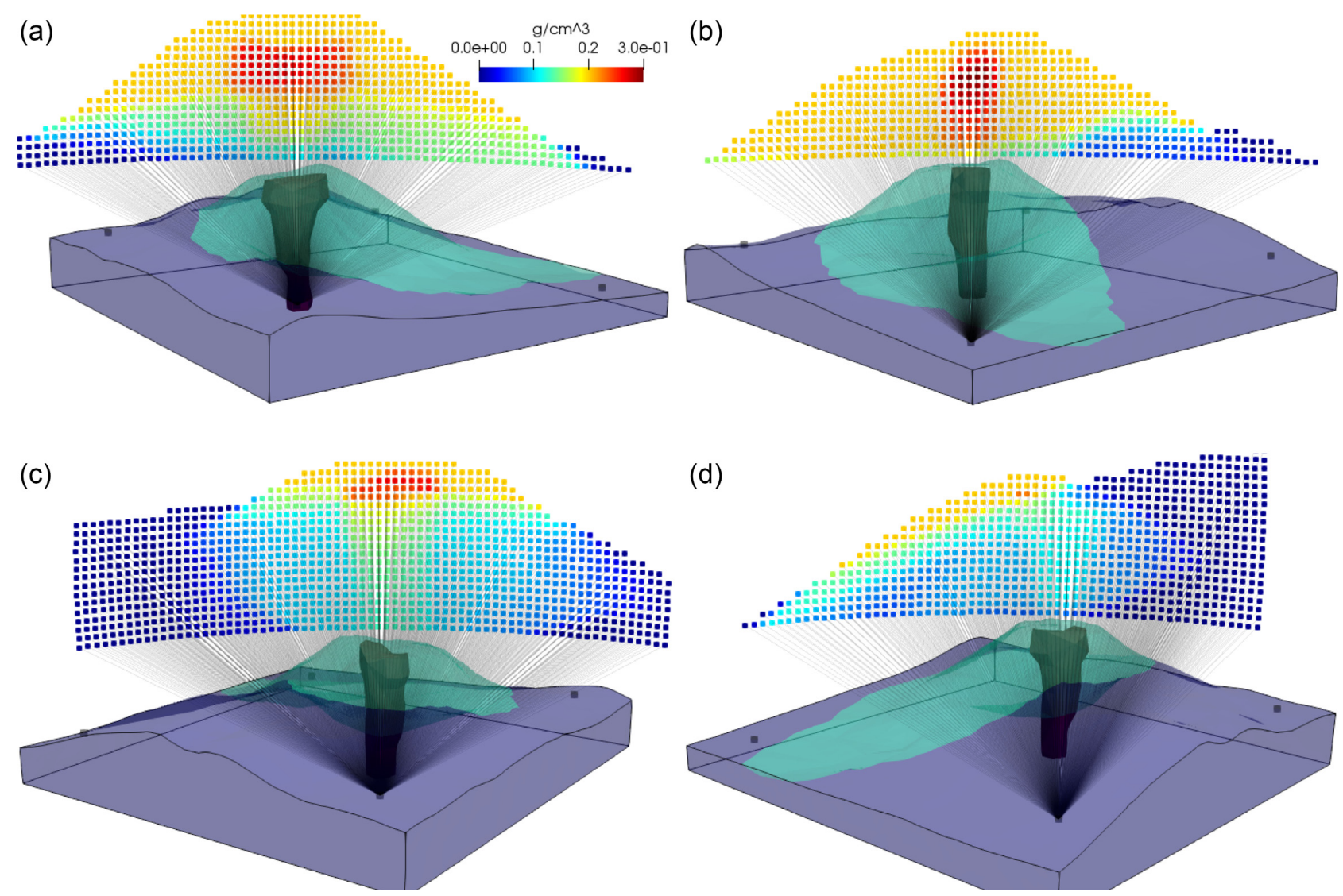

Figure 4. The synthetic muography data without noise added. Each panel shows a different perspective view looking from a single detector through the model: (a) the southwest detector, (b) the southeast detector, (c) the northwest detector, (d) the northeast detector. For each panel, the data are plotted $3 \mathrm{~km}$ away from the detector along their respective lines of sight. Each coloured point represents the average density value along a single one degree solid angle bin. The same colour scale is used for each panel and shows average density relative to $1.6 \mathrm{~g} \mathrm{~cm}^{-3}$ on the range $[0,0.3] \mathrm{g} \mathrm{cm}^{-3}$. The opaque black lines are drawn connecting the detector and each coloured data point to help aid the visualization.

challenges. Hence, we used an approximate noise model that captures the required flavour:

$\sigma=0.025+\frac{0.07}{1+e^{40(\hat{X}-0.02)}}+\frac{0.05}{1+e^{8(0.9-\hat{X})}}$,

where

(1) $\sigma$ is the statistical uncertainty

(2) $\hat{X}$ is the integrated absolute density (mass) remapped on $[0,1]$.

To arrive at this noise model, we manually fit an equation of the form in eq. (21) to the lower envelope of statistical uncertainties calculated for one particular set of muographic measurements from Puy de Dôme (see Fig. 5). Those values were arrived at using the simulation methods of Niess et al. (2018a,b). This noise model captures two essential characteristics. First, for solid angle bins that pass through smaller lengths of the Earth, for example those that are approximately tangential to the topography surface, the transmitted muon flux at the detector depends weakly on the density and the statistical errors affecting the measurements are magnified. Second, at large depths there are far fewer transmitted muons and the statistical errors again increase.

The integrated densities for our simplified synthetic model are significantly lower than those in the true Earth because we ignored density contrast between near-horizontal units. According to the work of Portal et al. (2016), the true Earth at Puy de Dôme is

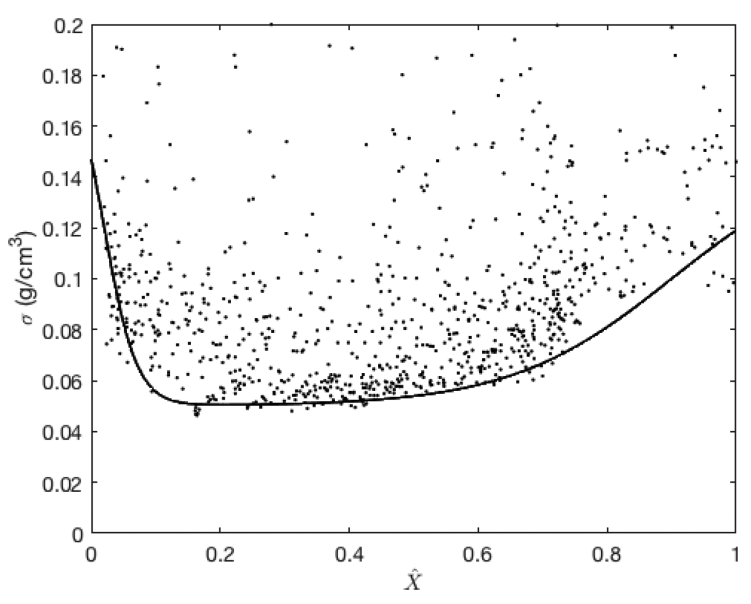

Figure 5. A plot of our approximate noise model and the data used to arrive at that model: $\sigma$ is statistical uncertainty and $\hat{X}$ is integrated absolute density. The black dots are the data used to develop our noise model. The solid line is our noise model, eq. (21), multiplied by two.

thought to have density values roughly between 1.6 and $2.5 \mathrm{~g} \mathrm{~cm}^{-3}$ in those units. Consequently, to achieve an appropriate estimate of the statistical uncertainties for our synthetic example, we used half the estimate fit to the measured data, leading to a somewhat optimistic treatment of the uncertainties. Several aspects of the detector used 

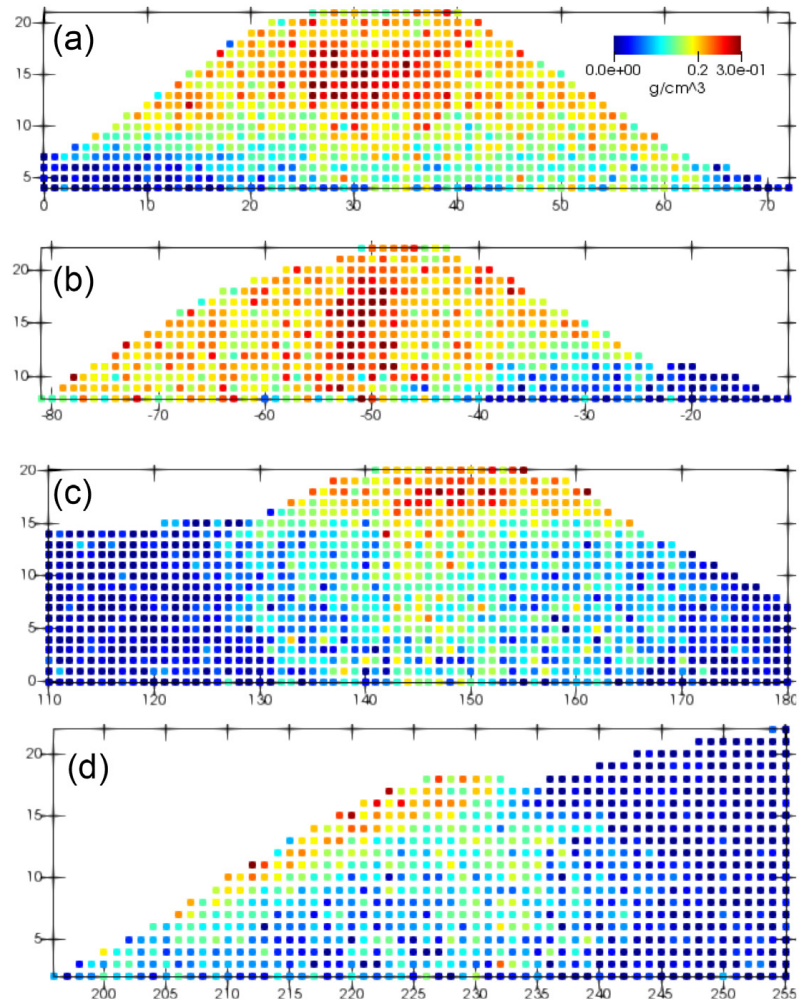

Figure 6. The synthetic muography data with noise added for each detector: (a) the southwest detector, (b) the southeast detector, (c) the northwest detector, (d) the northeast detector. Instead of the 3-D perspective views used in Fig. 4, here we simply provide 2-D plots of the data with the azimuthal angle (declination) on the horizontal axis and the polar angle on the vertical axis. The polar angle is zero at horizontal and positive above. The same colour scale is used for each panel and shows average density relative to $1.6 \mathrm{~g} \mathrm{~cm}^{-3}$ on the same range as in Fig. 4: $[0.0,0.3] \mathrm{g} \mathrm{cm}^{-3}$.

in the real survey on Puy de Dôme could have been improved, its size for example, and longer exposure time would also help reduce uncertainty levels; therefore we feel our approach here is not unrealistic.

We generated noise from normal distributions with zero mean and standard deviations calculated following eq. (21). Again, we made sure that eq. (20) was obeyed. Fig. 6 shows the noisy muography data.

\subsection{The inversion mesh}

To invert, we used a different mesh to that used in the forward modelling above so that the inversion mesh did not unfairly bias the results towards a favourable outcome. The inversion mesh was generated by removing all internal model features in the PLC (the model in Fig. 1) so that only the surfaces on the boundary of the model remained, including the topographic surface. The resulting PLC was then meshed using TetGen. Hence, the mesh used for inversion does not contain tetrahedral faces that honour the surfaces in the true synthetic model. To generate the volumetric mesh of tetrahedra, we supplied TetGen with a maximum tetrahedron volume constraint of $4 \times 10^{4} \mathrm{~m}^{3}$ in a $1000 \mathrm{~m}$ by $1000 \mathrm{~m}$ area surrounding the conduit, and a volume constraint of $4 \times 10^{5} \mathrm{~m}^{3}$ outside of that region. This provided a mesh with 27671 tetrahedral cells. (a)

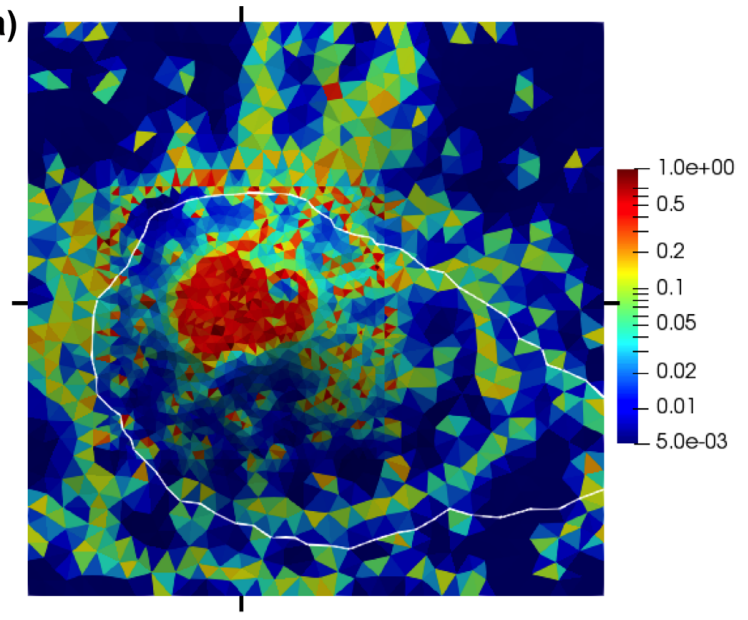

(b)

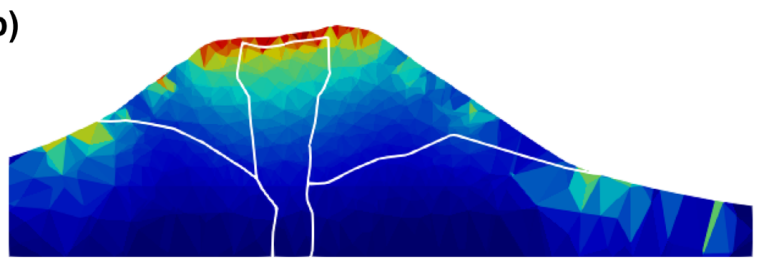

(c)

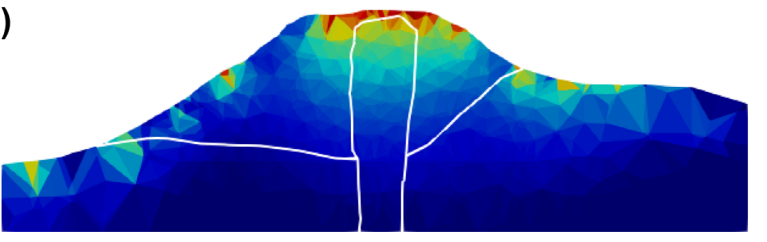

Figure 7. Overhead view (a) and cross-sections (b and c) through the inversion mesh showing gravity sensitivity information following eq. (22). The cross-sections are those used in Fig. 2. The small black marks around the outline of panel (a) indicate the locations of the cross-sections. The same logarithmic colour scale is used for each panel. The white line in (a) indicates the outcrop of the volcanic dome unit in the true model. The white lines in (b) and (c) indicate the outlines of the volcanic dome and conduit units in the true model.

\subsection{Sensitivity analysis}

Figs 7 and 8 show summed sensitivities for each cell in the inversion mesh. For each cell we calculated the sum

$s_{j}=\frac{\sqrt{\sum_{i=1}^{N} G_{i j}^{2}}}{v_{j}}$,

where $v_{j}$ is the volume of the $j$ th cell. We then normalized the $s_{j}$ values so they lay on $[0,1]$ before plotting. Fig. 7 shows the fall-off in gravity sensitivity away from the observation locations. Fig. 8 clearly shows the limited vertical resolution of the muography survey, which can only see upwards from the detector elevations. The lowest detector is at elevation $931 \mathrm{~m}, 131 \mathrm{~m}$ above the bottom of the modelling volume (which is at elevation $800 \mathrm{~m}$ ).

We wish to adequately image the bottom-most extent of the conduit within the modelling mesh. The conduit is approximately $110 \mathrm{~m}$ wide at that location. The vertical gravity response of a spherical mass with radius $110 \mathrm{~m}$, anomalous density $0.5 \mathrm{~g} \mathrm{~cm}^{-3}$, measured at a height of $660 \mathrm{~m}$ above the centre of the spherical mass, is $0.043 \mathrm{mGal}$. Hence, the signal from the bottom of the conduit is just detectable above the noise level added to the gravity data. 
(a)

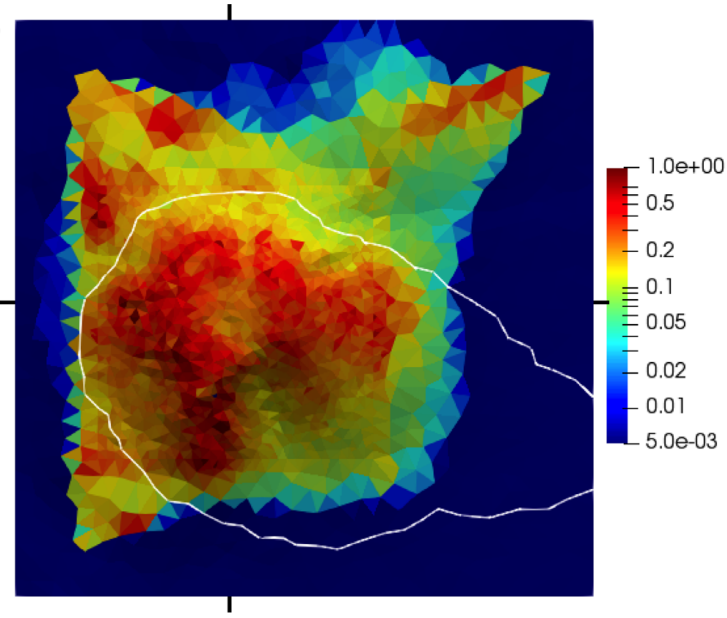

(b)

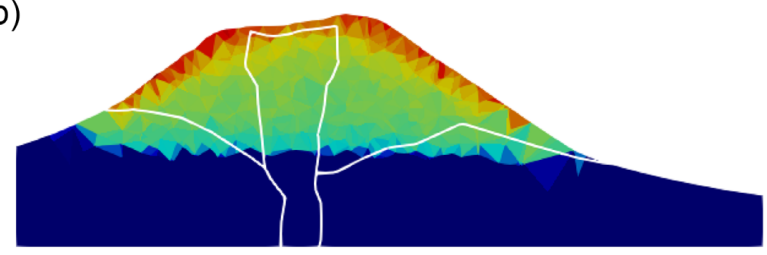

(c)

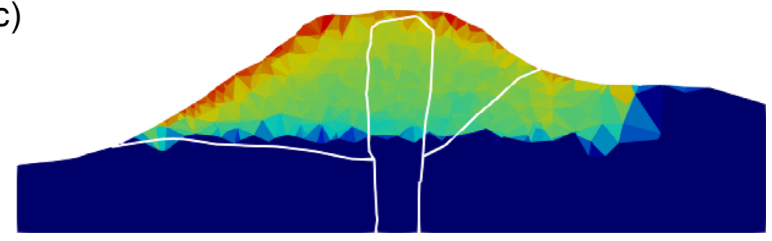

Figure 8. Overhead view (a) and cross-sections (b and c) through the inversion mesh showing muography sensitivity information following eq. (22). The cross-sections are those used in Fig. 2. The same logarithmic colour scale is used for each panel. The white overlays are as described for Fig. 7.

\subsection{Independent inversion results}

Here we show the results of inverting the two data sets separately. In these and all other inversions, we set $\alpha_{s}=0$ and $\alpha_{m}=1$ in eq. (6), thereby removing the smallness term, because there is no prior information suggesting the recovered density models should be small, but keeping the smoothness term. We set the target misfit(s) equal to the number of data, $N$, and the inversions stopped when

$\frac{\left|\Phi_{d}-N\right|}{N} \leq \epsilon$,

with $\epsilon=0.05$.

Figs 9 and 10 show the results of inverting the gravity data: Fig. 9 shows predicted and residual data maps; Fig. 10 shows the recovered density model. Similarly, Figs 11 and 12 show the results of inverting the muography data. Data maps are not shown for any future inversions in this manuscript because the results are comparable in character to those in Figs 9 and 11, that is, the predicted data responses match the synthetic observed data responses well, and the residual maps are random with no spatially correlated features, other than as discussed below.

In Fig. 9, the only significant spatially coherent high magnitude residuals are near the summit of the dome. Those likely result from the proximity of the gravity data to the sharp density discontinuity between the top of the conduit and the surrounding unit, and the (a)

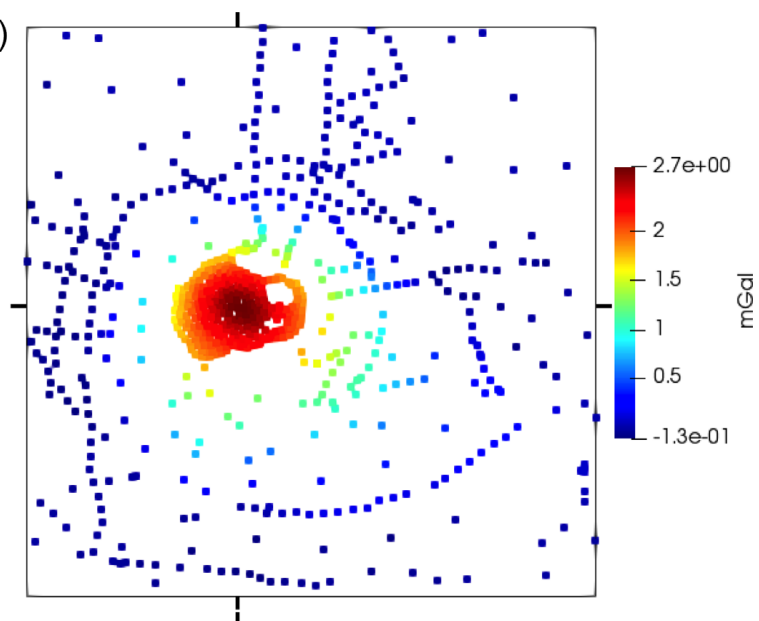

(b)

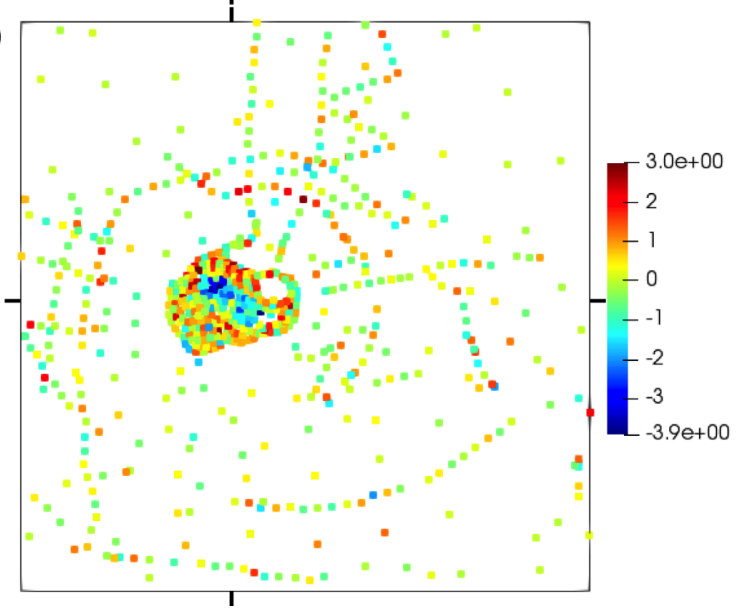

Figure 9. Predicted (a) and normalized residual (b) data maps for independent inversion of our synthetic gravity data.

inability of the inversion mesh to reproduce the required sharp features with the finite dimension cells used.

We indicate relative density contours at 0.1 and $0.3 \mathrm{~g} \mathrm{~cm}^{-3}$ on the images of the recovered models (Figs 10 and 12). Those contour values lie between the relative densities for the three values in the true model $\left(0.0,0.2\right.$ and $\left.0.5 \mathrm{~g} \mathrm{~cm}^{-3}\right)$ and are provided to help readers compare all inversion results in this manuscript when density colour scales change. The black contours should not be compared against the white overlays to assess each inversion result! Rather, the contours provide a reference to help compare one inversion result to another. Furthermore, we note that there is no one specific imaging target here; rather, we are looking to accurately image the entire structure of the volcano, including the depth extent, geometry and densities of both volcanic dome and conduit units.

The independent inversion results are consistent with the sensitivity analysis provided above. The gravity inversion result in Fig. 10 has a high amplitude positive density anomaly at the location of the conduit but the recovery of that unit is poor at depth, despite the applied distance weighting. Most of the largest positive densities are inside the region corresponding to the volcanic dome but there are significant positive densities at depth where no anomalous density exists in the true synthetic model: this inversion result would likely suggest to an interpreter a deeper volcanic dome unit than in the true model.

The muography inversion result in Fig. 12 also has a high amplitude positive density anomaly at the location of the conduit but 
(a)

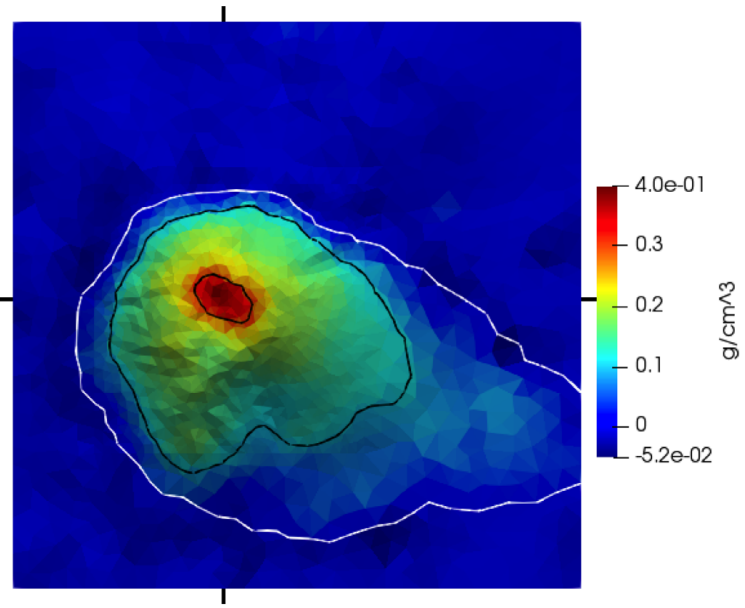

(b)

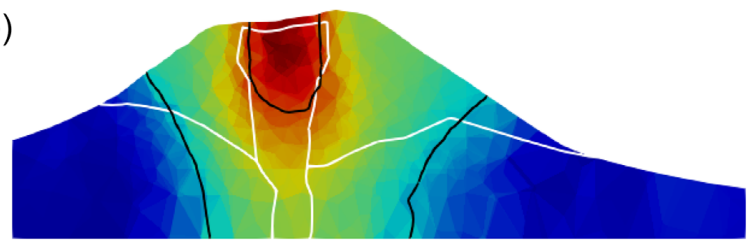

(c)

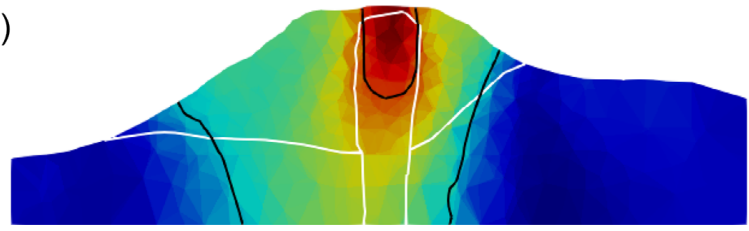

Figure 10. The density model recovered from independent inversion of our synthetic gravity data. The densities shown are relative to $1.6 \mathrm{~g} \mathrm{~cm}^{-3}$. The white overlays are as described for Fig. 7. The black lines in all panels indicate density contours at 0.1 and $0.3 \mathrm{~g} \mathrm{~cm}^{-3}$, corresponding to intermediate relative densities between the background and dome units, and between the dome and conduit units (see Table 1). We emphasize that the black contours should not be compared against the white overlays to assess each inversion result; rather, the contours provide a reference to help compare one inversion result to another.
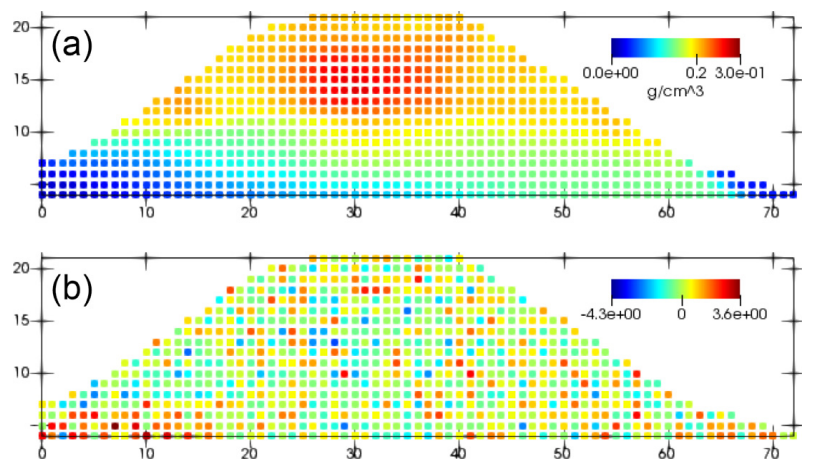

Figure 11. Predicted (a) and normalized residual (b) data maps for independent inversion of our synthetic muography data, plotted as in Fig. 6. Here we show only the maps for the first muon detector, although all data were inverted; the maps for the other detectors are comparable in character to these. (a)

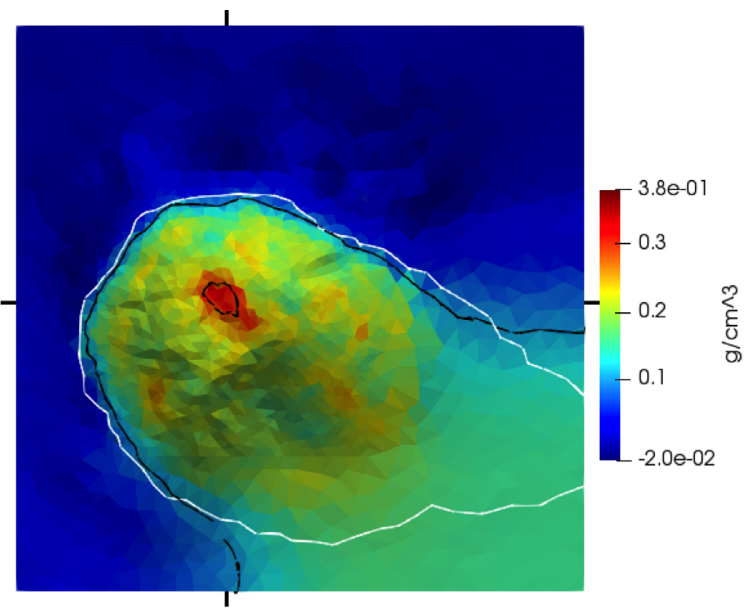

(b)

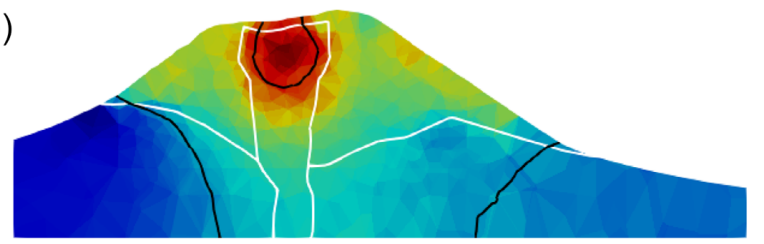

(c)

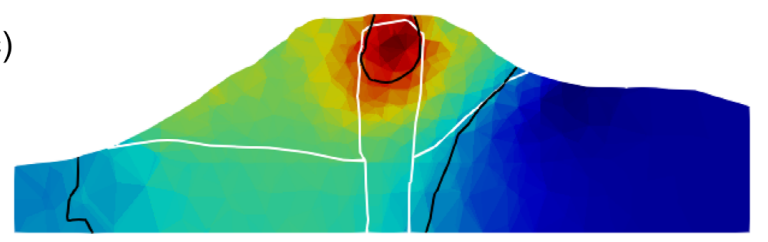

Figure 12. The density model recovered from independent inversion of our synthetic muography data. The densities shown are relative to $1.6 \mathrm{~g} \mathrm{~cm}^{-3}$. The white and black overlays are as described for Figs 7 and 10.

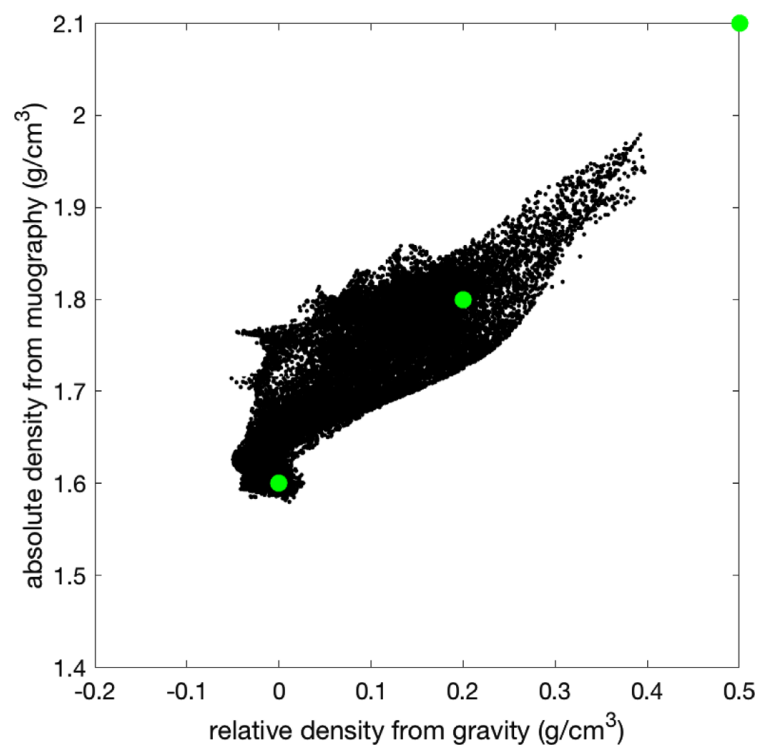

Figure 13. A cross-plot of the density values in the recovered models from independent gravity and muography inversion. Each black point represents density values taken from both models for a single cell in the mesh. Larger green circles indicate the values in the true model. 
places most of the largest positive densities at higher elevations than for the gravity inversion. The muography inversion recovered the volcanic dome unit better than the gravity data. However, the muography sensitivity falls to zero at a similar depth to the bottom of the volcanic dome unit and one must accept that any material below that depth is controlled only by the regularization. The muography inversion is unable to recover the correct background density, which is completely expected considering the complete lack of sensitivity at depth (see Fig. 8).

In Fig. 13 we show a cross-plot of the density values in the recovered models from independent gravity and muography inversion. These suggest a rough linear trend between the true values but there is a high degree of scatter. It is a common result that unconstrained, independent inversions are not able to adequately reproduce expected physical property relationships like this. In this case, it is predominantly a consequence of the different sensitivities of the two data types and of the smoothing regularization employed. One hopes that by jointly inverting the two data sets, such cross-plots will display a tighter linear relationship between the two models.

\subsection{Joint inversion for a single density model for both data types}

\subsubsection{Counteracting the sensitivity decay}

For joint inversion, when a single density model is inverted for, as the causative distribution for both data types, it is not immediately clear what weighting strategy to use to counteract the distance decay of the data sensitivity kernels. There is only one model and we could apply a distance weighting using the gravity observation locations, the muon detector locations, or a combination of the two. For brevity, we do not show the results, but our experiments indicated that, just as one might expect, applying distance weighting using only the muon detector locations was ineffective at counteracting the decay of the gravity kernels and the joint inversion results were similar to those without any distance weighting applied. However, without distance weighting, the joint inversions still did a reasonable job of pushing the dense material to depth, which perhaps counters experience with most gravity data collected on an approximately horizontal plane above a target: here we have gravity data collected across a topographic surface that essentially wraps around half of the volume of interest, so some sensitivity to depth can be expected. However, significant improvements were obtained by applying distance weighting, and using the combined gravity and muography observation locations provided similar results to using the gravity observation locations alone. Hence, we used distance weighting using the combined locations in all of our joint inversions that follow. To clarify, when jointly inverting, observation locations for both gravity and muography were included in the summation in eq. (7).

\subsubsection{The effect of the relative density offset on joint inversion results}

To aid in the comparison of our joint inversion results in the remainder of this manuscript, we now introduce two new terms. The 'offset estimate' is the value suggested by any particular joint inversion method, or by prior information before an inversion. This estimated value is subtracted from the observed muography data, either during or before an inversion, to attempt to correct for the density offset; refer to eq. (8). The 'offset remainder' is calculated as the actual relative offset between the two density distributions

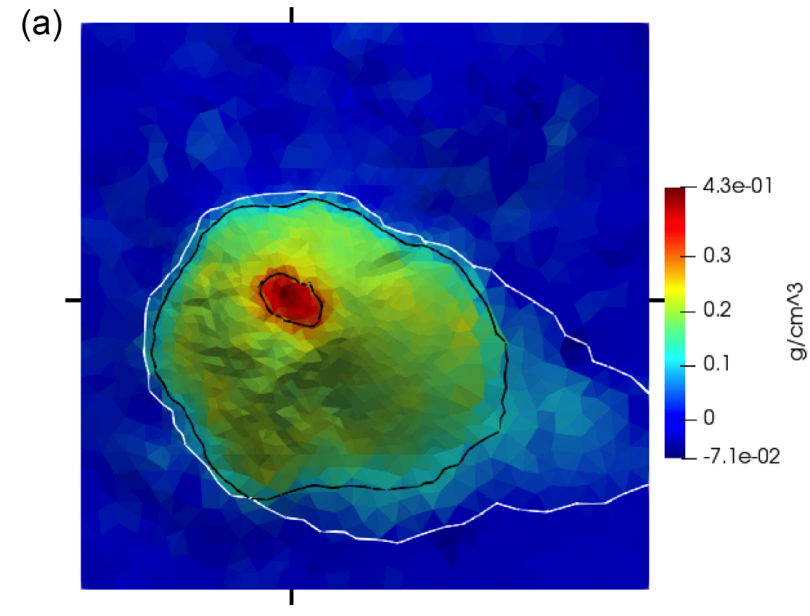

(b)

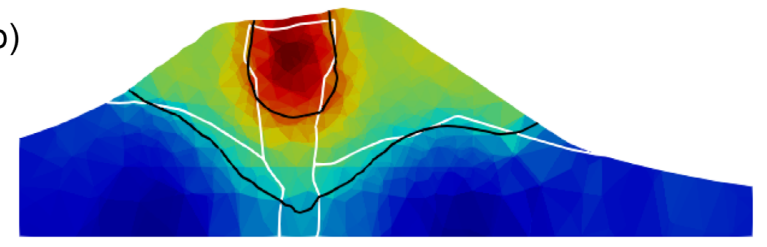

(c)

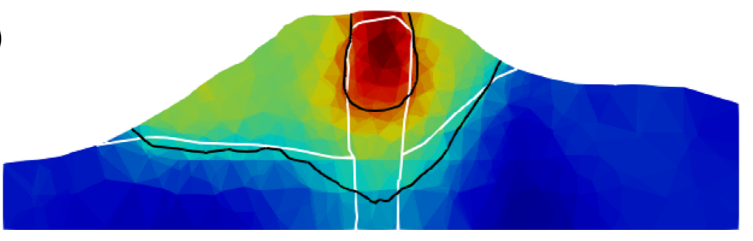

Figure 14. The density model recovered from joint inversion for a single density model and offset remainder of $0 \mathrm{~g} \mathrm{~cm}^{-3}$. The densities shown are relative to $1.6 \mathrm{~g} \mathrm{~cm}^{-3}$.

sensed by the gravity and muography data $\left(1.6 \mathrm{~g} \mathrm{~cm}^{-3}\right.$ for our scenario) minus the offset estimate; that is, it represents any offset that remains after the inversion attempts to correct for the offset (that correction not being perfect in general). Negative values of the offset remainder mean the joint inversion method overestimates the offset (leaving a net negative offset remainder); positive values mean the joint inversion method underestimates the offset.

Fig. 14 shows the joint inversion result using the formulation in eq. (3), and the standard misfit function in eq. (4), when the relative offset of $1.6 \mathrm{~g} \mathrm{~cm}^{-3}$ was removed exactly before inversion; the offset remainder is therefore $0.0 \mathrm{~g} \mathrm{~cm}^{-3}$, which is the ideal goal of any joint inversion applied to these two data types. There is an improved recovery of the depth extent of the volcanic dome unit compared to the independent inversion results. The conduit is clearly indicated as a positive density high within the volcanic dome unit and there is a slight indication of the conduit at the far depth extent of the mesh: although the densities there are not high enough to suggest conduit material, the dip of the lower black contour in Fig. 14 could suggest to an interpreter an extension of the conduit to depth, particularly taking into account the low resolution at these depths for the geophysical data involved and the smearing effect of these minimum-structure style inversions. We take the result in Fig. 14 to be the best possible for these data sets and we compare all our other joint inversion results to those.

As we mentioned previously, the hope was that joint inversion would better recover the true model at depth, below the coverage of the muography data. With that in mind, the joint inversion result 
(a)

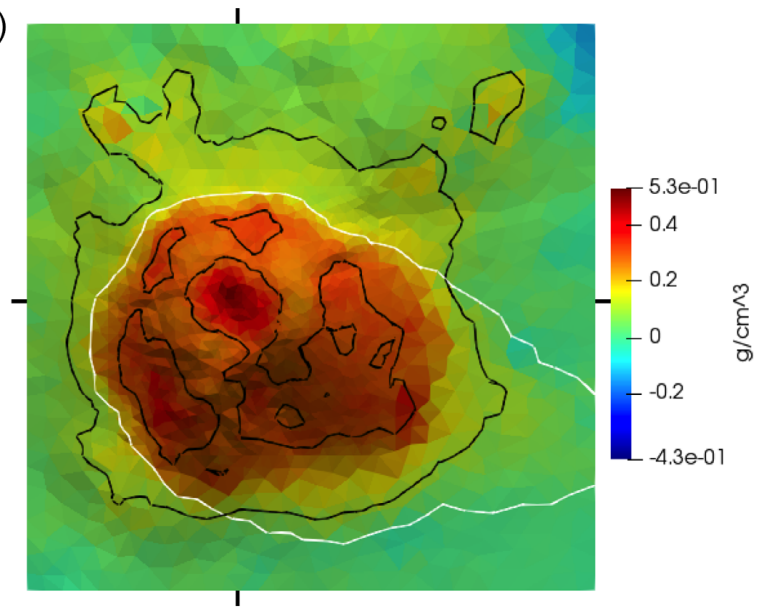

(b)

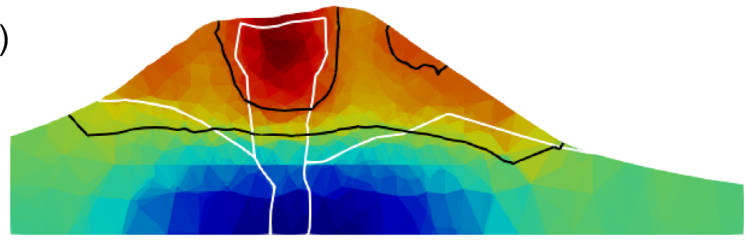

(c)

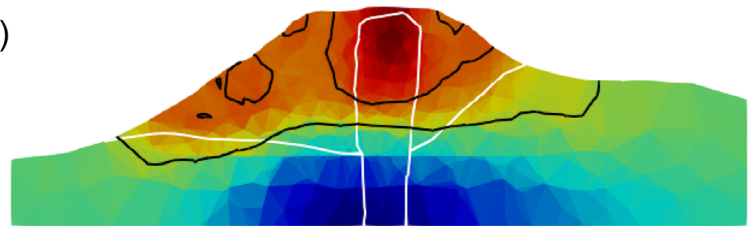

Figure 15. The density model recovered from joint inversion for a single density model and offset remainder of $+0.1 \mathrm{~g} \mathrm{~cm}^{-3}$. The densities shown are relative to $1.6 \mathrm{~g} \mathrm{~cm}^{-3}$.

in Fig. 14 has indeed improved the recovery of the true model below the coverage of the muography data in two ways. First, the independent inversion results in Figs 10 and 12 contain significant positive densities at depth where no anomalous density exists in the true synthetic model. Second, while the location and width of the conduit at depth is difficult to interpret from the smooth inversion results, one would likely interpret a much wider conduit from the independent gravity result in Fig. 10 than from the joint inversion result in Fig. 14. Furthermore, when comparing the black density contour overlays, the independent gravity inversion suggests a location for the conduit at depth that is somewhat shifted laterally from the true location, whereas the joint inversion indicates the correct lateral position.

To assess the effect of the relative offset, we jointly inverted, again using eqs (3) and (4), with various different offset estimates used to shift the muography data before inversion. To demonstrate the effect on the results for small and moderate offset remainders, we show the results for offset remainders of \pm 0.03 and $\pm 0.1 \mathrm{~g} \mathrm{~cm}^{-3}$ in Figs 15 through 18 . These values represent 6 per cent and 20 per cent of the density range in the true synthetic model $\left(0.5 \mathrm{~g} \mathrm{~cm}^{-3}\right)$.

When the offset remainder is positive, we have the scenario where an offset estimate was too low, meaning an insufficient offset correction has been applied by subtracting too small a constant value from the muography data. This could even correspond to a situation where the original data has been used in the inversion without any attempt at offset correction. The adjusted muography data then relates to a higher background density value than the gravity data. For (a)

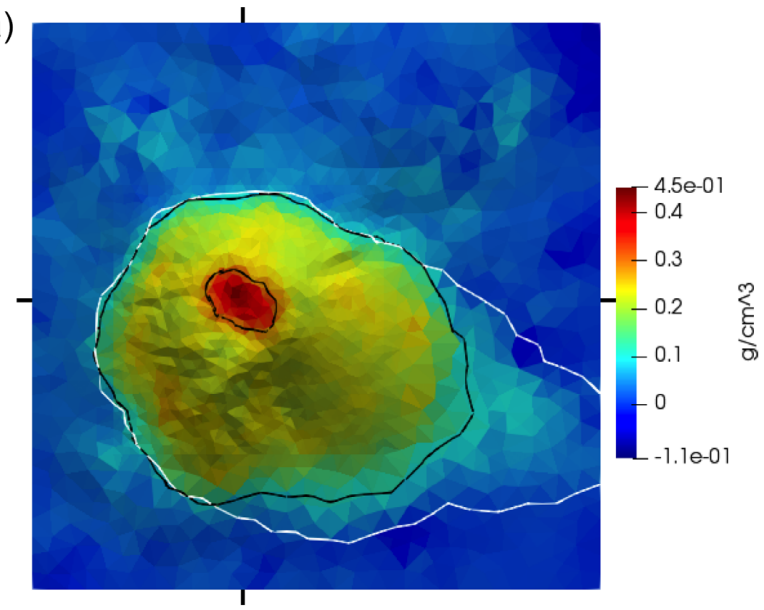

(b)

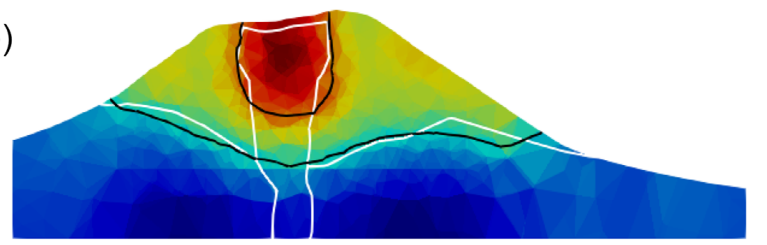

(c)

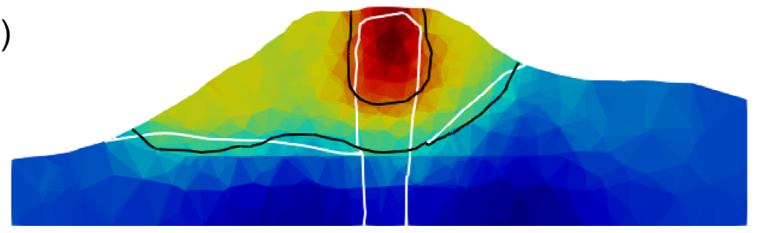

Figure 16. The density model recovered from joint inversion for a single density model and offset remainder of $+0.03 \mathrm{~g} \mathrm{~cm}^{-3}$. The densities shown are relative to $1.6 \mathrm{~g} \mathrm{~cm}^{-3}$.

the inversions with a positive offset remainder, Figs 15 and 16, there is less dense material recovered at depth: the density is effectively pushed upwards.

When the offset remainder is negative, we have the scenario where an offset estimate was too low, meaning too large a correction has been applied by subtracting too large a constant value from the muography data. The adjusted muography data then relates to a lower background density value than the gravity data. For the inversions with a negative offset remainder, Figs 17 and 18, there is more dense material recovered at depth: the density is effectively pushed downwards.

We explain these experimental observations by accepting that the gravity inverse problem is more non-unique than the muography problem and, hence, the gravity data has less power to influence the joint inversion. However, the model volume is only sensitive to the muography data in the top half of the model, above the muon detector locations. With a positive offset remainder, the offset has been underestimated and the muography data is higher than it should be; the muography data forces the recovery of higher densities in the top half of the model, and the inversion must then place lower densities at depth to fit the gravity data. For a negative offset remainder, the opposite occurs.

There are important differences in these results when even a small offset remainder occurs. In Fig. 16 there is very little that might suggest to an interpreter the extension of the conduit to depth; in Fig. 17 the volume of significant densities recovered at depth is significantly wider than in Fig. 14. Hence, obtaining accurate 
(a)

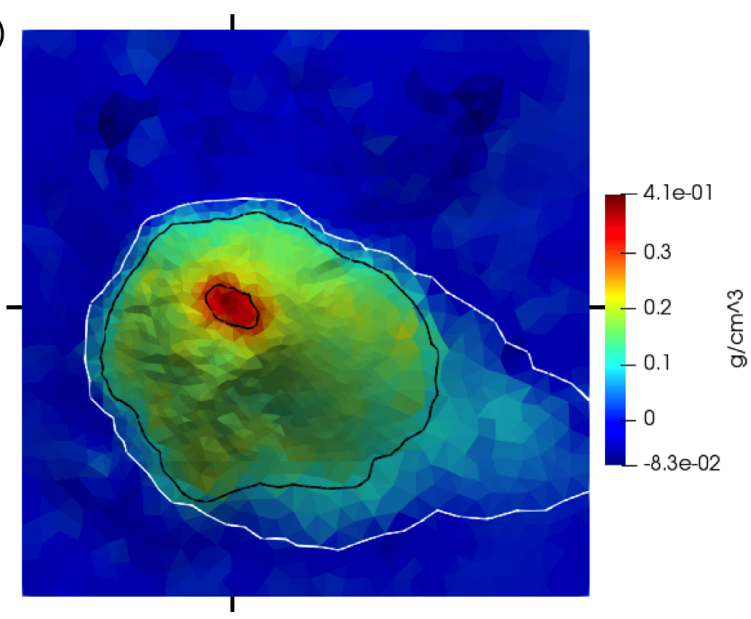

(b)

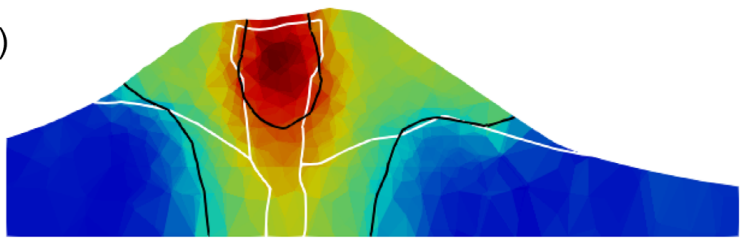

(c)

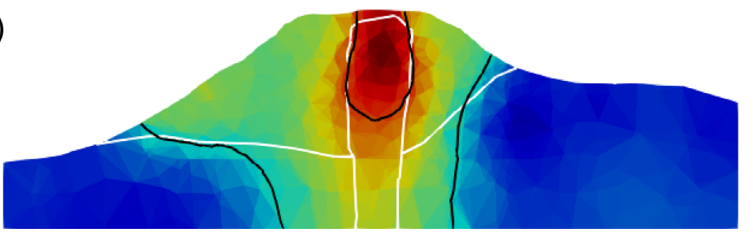

Figure 17. The density model recovered from joint inversion for a single density model and offset remainder of $-0.03 \mathrm{~g} \mathrm{~cm}^{-3}$. The densities shown are relative to $1.6 \mathrm{~g} \mathrm{~cm}^{-3}$.

prior information about the relative density offset is critical when performing joint inversions using this rather naive joint inversion approach.

\subsubsection{Automatic least-squares minimization for the offset}

Here we inverted using our LSM method to determine an appropriate offset, using the formulation in eq. (3) with the misfit defined in eq. (12). The true offset is $1.6 \mathrm{~g} \mathrm{~cm}^{-3}$, the inversion provided an offset estimate of $1.61 \mathrm{~g} \mathrm{~cm}^{-3}$, giving an offset remainder of $-0.01 \mathrm{~g} \mathrm{~cm}^{-3}$. We ran several other inversions, with different true offsets between the density distributions used to calculate the muography and gravity data responses, and all inversions had offset remainders of $-0.01 \mathrm{~g} \mathrm{~cm}^{-3}$; that is, the offset was overestimated by the same amount regardless of the true offset.

Fig. 19 shows the model recovered from one of these inversions. The LSM method provided an acceptable estimate of the offset but we have shown above that even a small offset remainder can lead to possible misinterpretations of the Earth's subsurface from subsequent joint inversions. In this case, however, the issue is minor. Specifically, in Fig. 19 one might interpret a slightly larger width for the conduit at depth than in the true synthetic model, or one might interpret a slightly deeper depth for the volcanic dome unit, but these misinterpretations are not particularly significant.

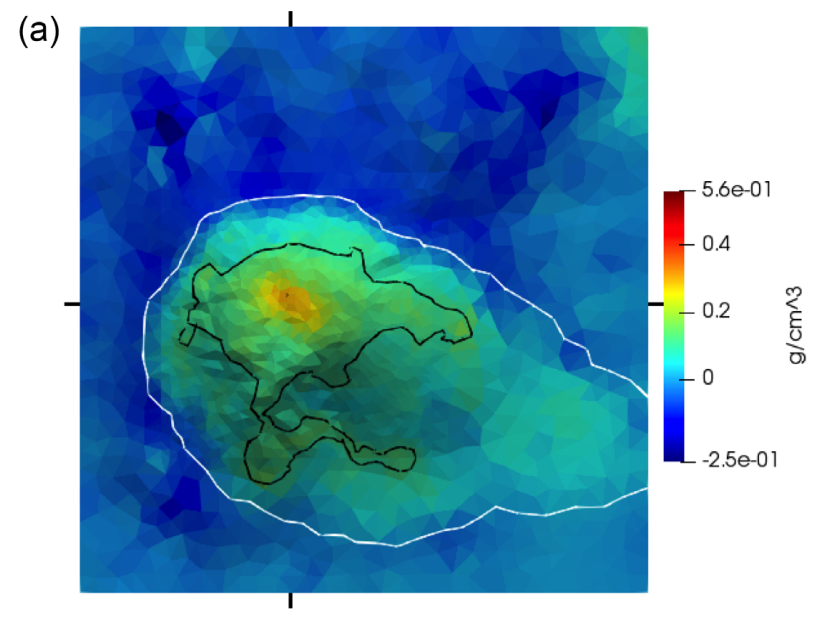

(b)

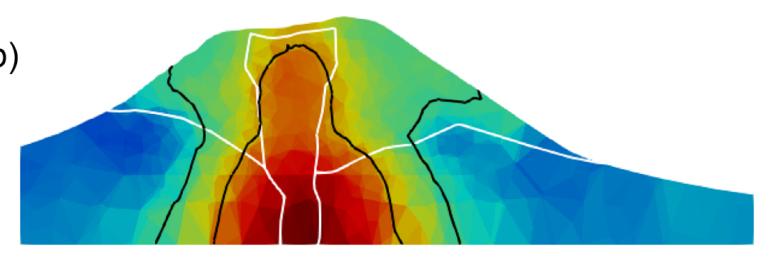

(c)

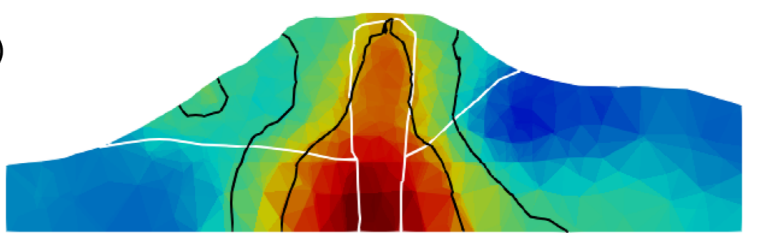

Figure 18. The density model recovered from joint inversion for a single density model and offset remainder of $-0.1 \mathrm{~g} \mathrm{~cm}^{-3}$. The densities shown are relative to $1.6 \mathrm{~g} \mathrm{~cm}^{-3}$.

\subsubsection{Additional offset parameter in the inversion}

Here we incorporated an additional inversion parameter for the offset, following eq. (13), with the standard misfit function in eq. (4). We performed several inversions with different values for the $\alpha_{c}$ parameter in eq. (13) over several orders of magnitude. Setting an appropriate weight for the $\alpha_{c}$ parameter was critical but simple to achieve. Fig. 20 shows a plot of the recovered offset estimate, $c$, versus the $\alpha_{c}$ value. With too high a value for $\alpha_{c}$, large amplitude values of $c$ are penalized, resulting in a lower amplitude value recovered for $c$ than the true offset, and the recovered model displays similar but more severe issues to that in Fig. 15. With too low a value for $\alpha_{c}$, the value of $c$ is effectively unregularized and numerical issues could result. However, no such issues were seen in the inversions we ran, and with low enough values for $\alpha_{c}$, the inversions provided a reasonable offset estimate of $1.62 \mathrm{~g} \mathrm{~cm}^{-3}$, meaning they suggested removal of $1.62 \mathrm{~g} \mathrm{~cm}^{-3}$ from the muography data, giving an offset remainder of $-0.02 \mathrm{~g} \mathrm{~cm}^{-3}$. We do not show the recovered models with low enough values of $\alpha_{c}$ because they are intermediate between those in Figs 17 and 19 for which the offset remainders were -0.03 and $-0.01 \mathrm{~g} \mathrm{~cm}^{-3}$, respectively.

\subsection{Joint inversion for two density models and equal gradient coupling}

Here we formulated the joint inverse problem as in eq. (14) using the coupling measure in eq. (19) and the standard misfit function 
(a)

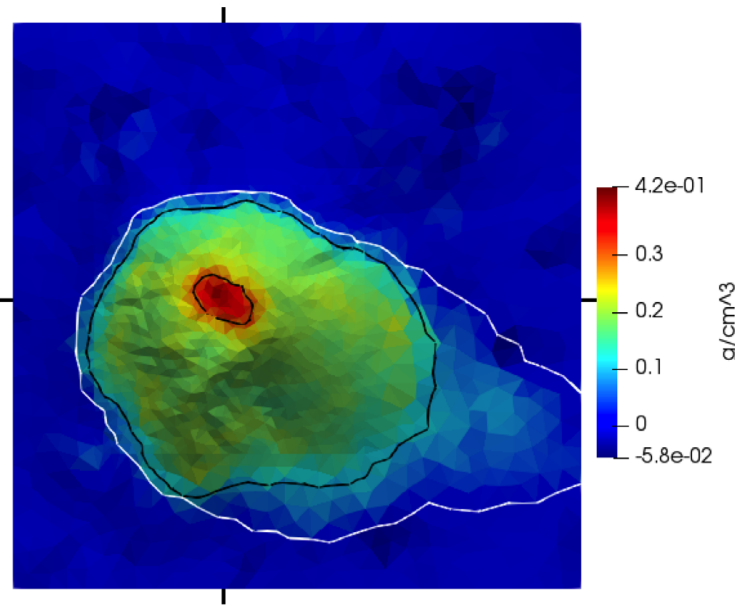

(b)

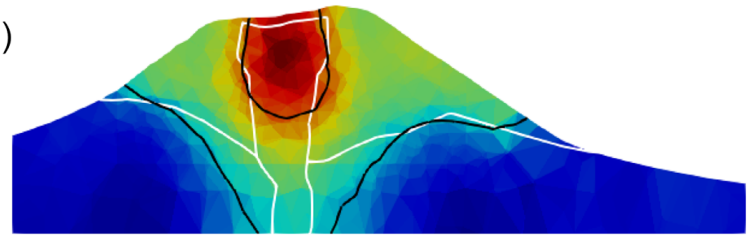

(c)

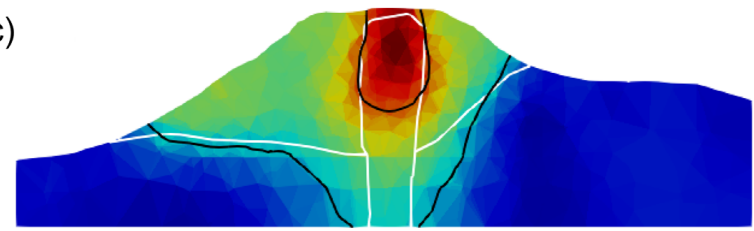

Figure 19. The density model recovered from joint inversion for a single density model and with the offset determined using our LSM method. The densities shown are relative to $1.6 \mathrm{~g} \mathrm{~cm}^{-3}$.

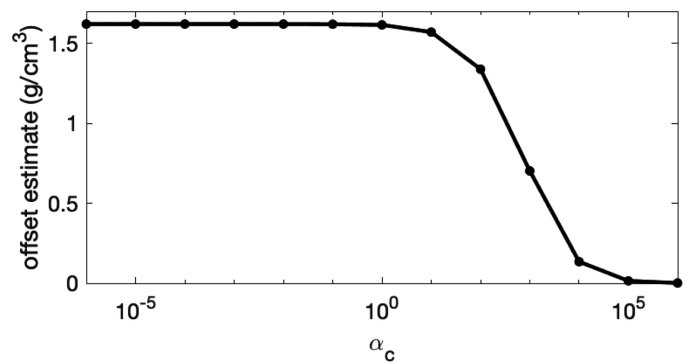

Figure 20. Recovered offset estimate versus $\alpha_{c}$ for joint inversion for a single density model and an additional offset parameter in the inversion. The black points indicate the data values from several joint inversions. A black line is drawn between the points.

in eq. (4). We performed several inversions with different values for the $\gamma$ parameter in eq. (14). Setting an appropriate weight for the $\gamma$ coupling weight was critical but not too difficult to achieve through a guess-and-check approach. With too low a value for $\gamma$, there is no coupling and the results mirror the independent inversion results. With a high enough value, the models are encouraged to have equal gradient and the cross-plot shows a more linear character. Hence, all practitioners must do is perform a few inversions with different $\gamma$ values across a fairly high range and compare the recovered models and cross-plots against the independent inversion results.

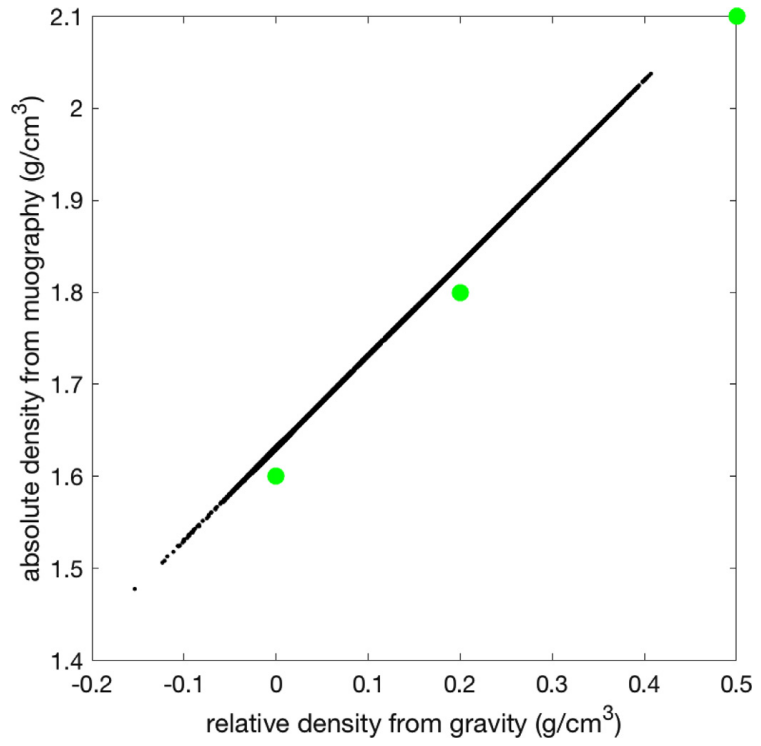

Figure 21. Cross-plots of the density values in the recovered models from joint inversion for two density models, equal gradient coupling with $\gamma=$ $1 \times 10^{6}$ in eq. (19). Each black point represents density values taken from both models for a single cell in the mesh. The larger green circles indicate the values in the true model.

Table 3. Summary of offset estimates and remainders recovered for our joint inversion tests. The offset estimate is the value suggested by an inversion, or by prior information before an inversion. The offset remainder equals the true offset $\left(1.6 \mathrm{~g} \mathrm{~cm}^{-3}\right)$ minus the offset estimate.

\begin{tabular}{lccc}
\hline Method & Figure & $\begin{array}{c}\text { Offset } \\
\text { estimate } \\
\left(\mathrm{g} \mathrm{cm}^{-3}\right)\end{array}$ & $\begin{array}{c}\text { Offset } \\
\text { remainder } \\
\left(\mathrm{g} \mathrm{cm}^{-3}\right)\end{array}$ \\
\hline No offset correction & 14 & 0.00 & 0.00 \\
$”$ & 15 & 1.50 & 0.10 \\
$"$ & 16 & 1.57 & 0.03 \\
$"$ & 17 & 1.63 & -0.03 \\
LSM & 18 & 1.70 & -0.10 \\
Additional parameter & 19 & 1.61 & -0.01 \\
Equal gradient & - & 1.62 & -0.02 \\
coupling & - & 1.63 & -0.03 \\
\hline
\end{tabular}

Fig. 21 shows a cross-plot of the recovered densities for an inversion with a relatively high value, $\gamma=1 \times 10^{6}$. A linear regression performed on the recovered densities (black points in Fig. 21) provided a slope of 1.00 and intercept of $1.63 \mathrm{~g} \mathrm{~cm}^{-3}$, meaning the inversion suggests removal of $1.63 \mathrm{~g} \mathrm{~cm}^{-3}$ from the muography data, corresponding to an offset remainder of $-0.03 \mathrm{~g} \mathrm{~cm}^{-3}$. We do not show the results for this inversion because they are comparable to those in Fig. 17.

To aid comparison of the various joint inversion methods applied to our scenario, Table 3 provides a summary of the offset estimates and remainders for all the joint inversion tests.

\section{DISCUSSION}

Before concluding, we discuss the various assumptions we made in our work so that our results and conclusions can be interpreted within an appropriate scope. Any extensions to this work to reduce these assumptions we leave for future work. Perhaps the most important assumption made in this work is that the relative density 
offset can be approximated to first order as a constant difference, although it may in fact vary. For gravity data, the appropriate background density for the Bouguer and terrain corrections is expected to be reasonably constant over relatively small distances representative of volcanic domes similar to Puy de Dôme, so the first-order approximation should be reasonably valid. Also, a future generation of muon detectors, and improved methodologies for the muography inverse problem, should significantly reduce the systematic uncertainties affecting muography data. For example, the work of Gómez et al. (2017), which characterizes the dependence of muon scattering as a function of the relative angle between the detector and the topography, suggests that a theoretical correction should be possible. We can therefore conclude that the approach developed in this manuscript, approximating the offset as a constant, should become even more suitable in the future.

Our 3-D synthetic volcano model was built from existing geological and geophysical information. However, we simplified the model somewhat to remove some extraneous features, but most of those features did not cause significant anomalies in the gravity or muography data. Also, we did not include any heterogeneity within the rock units. Gravity and muography data sense bulk properties of the Earth: gravity data are sensitive to the entire Earth but the sensitivities are somewhat diffusive, and muography data averages densities along solid angles, so smaller scale random density heterogeneity does not affect either type of data greatly.

We assumed that in a survey data scenario, the gravity data processing will have adequately removed any regional gravity components, that is, long wavelength responses from density outside the modelling volume. In practice, this might not be the case and there may be some effects on the recovered models and recovered offset estimates. However, if padding cells are added to the mesh to allow for deeper anomalous material then they may be able to deal with the long wavelength responses from density outside the modelling volume. Any coupling in the joint inverse problem should be limited to the central core volume of interest in the mesh, although the muography survey data will be insensitive to any density in the padding cells so this may not be necessary. A similar approach would be to only apply any joint coupling to mesh cells where both surveys have non-zero sensitivity.

If more padding cells were added, there would be a smaller percent volume of the mesh supported by the muography data compared to the gravity data. Our results might be expected to change if the percent volume of the mesh supported by the muography data were to increase or decrease. Future work should investigate the importance of the percent volume mesh support ratios for muography and gravity data.

This synthetic study used four muon detectors, ensuring adequate data coverage. It is likely that three appropriately located detectors would have been enough. However, the conclusions in this study may not apply for scenarios where there is only one or two detectors such that the muography survey cannot adequately resolve the volume of interest.

The noise model we used for the muography data was somewhat optimistic. We consider it a best case for muography, given our experiences with survey data, but statistical uncertainties could be reduced with an improved detector, a longer exposure time and improved data processing approaches. In our synthetic scenario, the noise levels on the gravity data and muography data were roughly comparable, qualitatively, and the results of our tests might be expected to change if one data set were to dominate the other. However, gravity data will always suffer from poor depth resolution, and muography data will likely always suffer from lateral resolution issues resulting from the limited number of detectors that can be placed in a practical survey campaign.

A smearing procedure (see Niess et al. 2018b) was performed when converting muon counts to average densities, which calculated statistics for a particular solid angle bin using muon count information from neighbouring bins as well. The large plateau in our noise model (see Fig. 5) is a result of this smearing procedure. Without that procedure, we would expect the error to increase at much lower integrated density values. Future work could investigate the effects of different muography noise levels on the behaviour of the joint inversion.

Another consequence of the smearing procedure is that in practice the muography errors are correlated. Modelling this correlation effect is a complicated undertaking and therefore we chose to neglect it in this work. To include it, we could have used a more general misfit expression in eq. (4) and included a full covariance matrix instead of only diagonal terms. However, improvements to muon detectors and increased exposure times can reduce the necessity to perform this smearing so, again, we consider that neglecting the muography data correlation is a best case for muography.

We considered the joint inversion results in Fig. 14 to be the best possible for these data sets. There is a clear indication of the bottom of the conduit but it is still poorly resolved. To resolve that feature, it is probably necessary to reduce the magnitude of the noise added to the synthetic data. We have modelled our noise based on realistic noise levels in survey data. Hence, we go no further in this study but we suggest that steps may need to be taken to reduce the noise levels for survey data collected in similar scenarios.

Finally, we note that the muon counts could be inverted directly, rather than using the average densities resulting from Monte-Carlo simulation procedures; this alternative approach could possibly reduce the errors introduced by the average density conversion. That would create a nonlinear inverse problem, which is a complication but is certainly possible. However, it would have little impact on the issue of how to handle the relative density offset. We leave a comparison of the linear and nonlinear muon tomography inverse problems to future work.

\section{CONCLUSION}

We have investigated various numerical approaches for formulating the joint inverse problem of inverting muography and gravity data. Specifically, we considered various approaches to ameliorate the complication that muography and gravity data are effectively responsive to different density quantities related by some unknown offset. We considered existing methods from previous works by other authors, and three new joint inversion methods that we developed. We applied those methods to a synthetic volcano imaging problem based on the Puy de Dôme volcano in the Central Massif region of France. We used unstructured meshes in our modelling to adequately honour the significant topography in that scenario but our conclusions below, relating to the joint inversion methods, apply to rectilinear meshes or other mesh-based representations of the Earth.

The naive approach of performing a joint inversion for a single density model, for both data types, and attempting to account for the relative offset using prior information is not recommended. Important differences were seen in the inversion results with even a small error in the recovered offset estimate, which could lead to possible misinterpretations about the Earth's subsurface. However, it may be possible that the sensitivity of such joint inversion results 
to the offset estimate varies for different Earth scenarios, data survey configurations and inversion parameters.

We developed a new approach for estimating the offset automatically in a joint inversion by reformulating the misfit function using a least-squares minimization argument. We named this the LSM method. This method provided an acceptable estimate of the offset for our test example and we recommend this approach be applied to similar scenarios to our synthetic.

Another joint inversion approach we considered, used previously by other authors, was to use a single density model but include an additional offset correction parameter in the inversion. This approach requires that an appropriate value is selected for a specific inversion parameter, a regularization weight associated with the offset correction parameter, but doing so is simple in practice. For our test example, this approach also provided a reasonable estimate for the offset and we recommend this approach be applied to similar scenarios to our synthetic in combination with the LSM method.

The joint inverse problem can also be posed using two density models, one for each data type, with an additional coupling term used to encourage some similarity between the two. We investigated a new coupling approach where the spatial gradients are encouraged to be equal. This introduces a linear term into the objective function so there is no increase in the computational requirements. This approach also requires that an appropriate value be selected for a specific inversion parameter, a joint coupling weight in this case, but doing so is again simple in practice. For our test example, this joint inversion approach provided a rough estimate of the offset and may have some merit if applied to similar scenarios. Hence, we suggest this approach be used in combination with the above two approaches but with some caution.

We made several important assumptions in our work, which we discussed in the previous section, and we stress that our results and final conclusion here should be interpreted within an appropriate scope. While our results and related conclusions are not likely to change if some of our assumptions are broken, future work should be performed to assess the importance of some of our larger assumptions. Future work could also investigate the stability of the recovered models and offset estimates for various parameters that tune the inversion, for example regularization weights and target misfit tolerance, $\epsilon$ in eq. (23), and when alternative regularization functionals are used.

Our experiments indicated that the most successful gravity and muography joint inversion method for the type of geological scenario tested is our LSM method. However, many of the approaches tested provided somewhat helpful results and may have some merit for general scenarios. Hence, our conclusion is that several of the methods should be applied to general problems and the results analysed for commonality.

\section{ACKNOWLEDGEMENTS}

We are grateful to Marina Rosas-Carbajal and Jiajia Sun for their detailed and helpful reviews that improved this manuscript. This research was financed by the French Government Laboratory of Excellence initiative $n^{\circ}$ ANR-10-LABX-0006, the Region Auvergne and the European Regional Development Fund. This is Laboratory of Excellence ClerVolc contribution number 322.

\section{REFERENCES}

Ambrosino, F. et al., 2015. Joint measurement of the atmospheric muon flux through the Puy de Dôme volcano with plastic scintillators and resistive plate chambers detectors, J. geophys. Res., 120(11), 7290-7307.

Barnoud, A., Cayol, V., Niess, V., Cârloganu, C., Lelièvre, P.G., Labazuy, P. \& Le Ménédeu, E., 2019. Bayesian joint muographic and gravimetric inversion applied to volcanoes, Geophys. J. Int., accepted.

Bijani, R., Lelièvre, P.G., Ponte-Neto, C.F. \& Farquharson, C.G., 2017. Physical property-, lithology- and surface-based joint inversion using Pareto multi-objective global optimization, Geophys. J. Int., 209, 730748.

Boivin, P., Besson, J., Briot, D., Deniel, C., Gourgaud, A., Labazuy, P. \& Langlois, E., 2017. Volcanology of the Chaine des Puys Massif Central Français, Parc naturel régional des Volcans d'Auvergne.

Cârloganu, C. et al., 2013. Towards a muon radiography of the puy de dôme, Geosci. Instrum. Method. Data Syst., 2, 55-60.

Davis, K. \& Oldenburg, D.W., 2012. Joint 3D inversion of muon tomography and gravity data to recover density, in ASEG Extended Abstracts 2012: 22nd Geophysical Conference, pp. 1-4, Brisbane, Australia.

Fregoso, E. \& Gallardo, L.A., 2009. Cross-gradients joint 3D inversion with applications to gravity and magnetic data, Geophysics, 74(4), L31-L42.

Gómez, H. et al., 2017. Forward scattering effects on muon imaging, $J$. Instrument., 12(12), P12018, doi:10.1088/1748-0221/12/12/P12018.

Günther, T., Rücker, C. \& Spitzer, K., 2006. Three-dimensional modelling and inversion of DC resistivity data incorporating topography - II. inversion, Geophys. J. Int., 166(2), 506-517.

Jourde, K., Gibert, D. \& Marteau, J., 2015. Improvement of density models of geological structures by fusion of gravity data and cosmic muon radiographies, Geosci. Instrum. Method. Data Syst., 4, 177-188.

Lelièvre, P.G. \& Farquharson, C.G., 2013. Gradient and smoothness regularization operators for geophysical inversion on unstructured meshes, Geophys. J. Int., 195(1), 330-341.

Lelièvre, P.G., Farquharson, C.G. \& Hurich, C.A., 2012. Joint inversion of seismic traveltimes and gravity data on unstructured grids with application to mineral exploration, Geophysics, 77(1), K1-K15.

Lelièvre, P.G. et al., 2018. FacetModeller: Software for manual creation, manipulation and analysis of 3D surface-based models, SoftwareX, 7, $41-46$.

Li, Y. \& Oldenburg, D.W., 2000. Joint inversion of surface and threecomponent borehole magnetic data, Geophysics, 65(2), 540-552.

Linsser, H., 1965. A generalized form of Nettleton's density determination, Geophys. Prospect., 13(2), 247-258.

Liu, Z., Bryman, D. \& Bueno, J., 2012. Application of muon geotomography to mineral exploration, in International Workshop on "Muon and Neutrino Radiography 2012", Clermont-Ferrand, France.

Moorkamp, M., Lelièvre, P.G., Linde, N. \& Khan, A., 2016. Geophysical Monograph Series, American Geophysical Union. Integrated Imaging of the Earth: Theory and Applications, John Wiley \& Sons, Inc., Hoboken, New Jersey.

Miller, G.L., Talmor, D., Teng, S., Walkington, N. \& Wang, H., 1996. Control volume meshes using sphere packing: Generation, refinement and coarsening, in Proceedings of the 5th International Meshing Roundtable, pp. 47-61, Sandia National Laboratories.

Morishima, K. et al., 2017. Discovery of a big void in Khufu's Pyramid by observation of cosmic-ray muons, Nature, 552, 386-390.

Nagamine, K., Iwasaki, M., Shimomura, K. \& Ishida, K., 1995. Method of probing inner-structure of geophysical substance with the horizontal cosmic-ray muons and possible application to volcanic eruption prediction, Nucl. Instrum. Method. Phys. Res., 356(2), 585-595.

Niess, V., Barnoud, A., Cârloganu, C. \& Le Ménédeu, E., 2018a. Backward Monte-Carlo applied to muon transport, Comput. Phys. Commun., 229, 54-67.

Niess, V., Barnoud, A., Cârloganu, C. \& Le Menedeu, E., 2018b. Methods and tools for transmission muography, in European Geosciences Union General Assembly, Vienna, Austria.

Nishiyama, R., Tanaka, Y., Okubo, S., Oshima, H., Tanaka, H.K.M. \& Maekawa, T., 2014. Integrated processing of muon radiography and gravity anomaly data toward the realization of high-resolution 3-D density 
structural analysis of volcanoes: case study of Showa-Shinzan lava dome, Usu, Japan, J. geophys. Res., 119, 699-710.

Nishiyama, R., Miyamoto, S., Okubo, S., Oshima, H. \& Maekawa, T., 2017. 3D density modeling with gravity and muon-radiographic observations in Showa-Shinzan lava dome, Usu, Japan, Pure appl. Geophys., 174(3), 1061-1070.

Okabe, M., 1979. Analytic expressions for gravity anomalies due to homogeneous polyhedral bodies and translations into magnetic anomalies, Geophysics, 44(4), 730-741.

Portal, A., 2015. Etude géophysique de la structure interne d'un dôme volcanique: le Puy de Dôme et son environnement (Chaîne des Puys, France), PhD thesis, Sciences de la Terre, Université Blaise Pascal, ClermontFerrand, France.

Portal, A. et al., 2013. Inner structure of the Puy de Dôme volcano: crosscomparison of geophysical models (ERT, gravimetry, muonic imagery), Geosci. Instrum. Methods Data Systems, 2, 47-54.

Portal, A., Gailler, L., Labazuy, P. \& Lénat, J., 2016. Geophysical imaging of the inner structure of a lava dome and its environment through gravimetry and magnetism, J. Volc. Geotherm. Res., 320, 88-99.

Procureur, S., 2018. Muon imaging: Principles, technologies and applications, Nucl. Instrum. Methods Phys. Res., 878, 169-179.

Rosas-Carbajal, M., Jourde, K., Marteau, J., Deroussi, S., Komorowski, J. \& Gibert, D., 2017. Three-dimensional density structure of La Soufrière de Guadeloupe lava dome from simultaneous muon radiographies and gravity data, Geophys. Res. Lett., 44, 6743-6751.

Schouten, D. \& Ledru, P., 2018. Muon tomography applied to a dense uranium deposit at the McArther River mine, J. geophys. Res., 123(10), 8637-8652.

$\mathrm{Si}, \mathrm{H} ., 2015$. TetGen, a delaunay-based quality tetrahedral mesh generator, ACM Trans. on Math. Softw., 41(2), doi:10.1145/2629697.

Si, H., 2017. TetGen: a quality tetrahedral mesh generator and a 3D delaunay triangulator. Available at: http://wias-berlin.de/software/tetgen/( last accessed November 2017).

Tanabashi, M. et al., 2018. Review of particle physics, Phys. Rev. D, 98, 030001 .

Tanaka, H.K.M. et al., 2010. Three-dimensional computational axial tomography scan of a volcano with cosmic ray muon radiography, J. geophys. Res., 115, B12332, doi:10.1029/2010JB007677.

Tontini, F.C., Graziano, F., Cocchi, L., Carmisciano, C. \& Stefanelli, P., 2007. Determining the optimal Bouguer density for a gravity data set: implications for the isostatic setting of the Mediterranean Sea, Geophys. J. Int., 169, 380-388.

Yamamoto, A., 1999. Estimating the optimal reduction density for gravity anomaly: a theoretical overview, J. Faculty Sci., 11(3), $577-599$. 